\title{
A dynamical interpretation of flutter instability in a continuous medium
}

\author{
Andrea Piccolroaz ${ }^{(*)}$, Davide Bigoni ${ }^{(*)}$ and John R. Willis ${ }^{(\circ)}$ \\ (*) Dipartimento di Ingegneria Meccanica e \\ Strutturale, Università di Trento, \\ Via Mesiano 77, I-38050 Trento, Italia \\ (॰) Department of Applied Mathematics and Theoretical Physics \\ Centre for Mathematical Sciences, Cambridge University \\ Wilberforce Road, Cambridge CB3 OWA U.K. \\ email: andrea.piccolroaz@ing.unitn.it; bigoni@ing.unitn.it; \\ J.R.Willis@damtp.cam.ac.uk
}

November 5, 2018

\begin{abstract}
Flutter instability in an infinite medium is a form of material instability corresponding to the occurrence of complex conjugate squares of the acceleration wave velocities. Although its occurrence is known to be possible in elastoplastic materials with nonassociative flow law and to correspond to some dynamically growing disturbance, its mechanical meaning has to date still eluded a precise interpretation. This is provided here by constructing the infinite-body, time-harmonic Green's function for the loading branch of an elastoplastic material in flutter conditions. Used as a perturbation, it reveals that flutter corresponds to a spatially blowing-up disturbance, exhibiting well-defined directional properties, determined by the wave directions for which the eigenvalues become complex conjugate. Flutter is shown to be connected to the formation of localized deformations, a dynamical phenomenon sharing geometrical similarities with the well-known mechanism of shear banding occurring under quasi-static loading. Flutter may occur much earlier than shear banding in a process of continued plastic deformation.
\end{abstract}

KEYWORDS: Green's function; elastoplastic materials; nonassociative flow rule; material instability; granular materials. 


\section{Introduction}

Several micromechanisms acting at a microscale during deformation of granular and rock-like materials involve Coulomb friction. As a consequence, the flow rule becomes nonassociative and the phenomenological rate elastoplastic constitutive equations for these materials become unsymmetric. Due to this lack of symmetry, two squares of the propagation velocity of acceleration waves or, in other words, two eigenvalues of the acoustic tensor, may become a complex conjugate pair. That this situation might correspond to a form of material instability particularly relevant in granular material was clear since J.R. Rice (1977) coined for it the term 'flutter instability', but neither examples of constitutive equations displaying this instability nor a mechanical interpretation for it were given at that time.

Consequently, research was initially focused on the determination of situations in which flutter was possible (see Bigoni, 2000; Loret et al., 2000 for reviews). In particular, it was shown that flutter instability may occur more often than one might expect, not satisfying any hierarchical relation to other instabilities (such as for instance shear banding or second-order work negativity), possibly at an early stage of a hardening process and typically triggered by noncoaxiality (of the flow rule or induced by elastic or plastic anisotropy). However, the problem of finding a mechanical interpretation for the instability remained almost completely unexplored [with the exceptions of Bigoni and Willis (1994) and Simões (1997), the former considering a very simple problem setting and the latter providing some numerical tests]. This has been a major problem retarding further progress in research since, though generically believed to correspond to a dynamically growing disturbance, only the knowledge of the precise mechanical features of the instability can permit its identification for real materials.

To shed light on this problem, a perturbative approach is developed in this article, following the methodology proposed by Bigoni and Capuani (2002; 2005) to investigate shear banding and other forms of material instabilities. In more detail, the analysis is limited in the present article to the loading branch 1 of an elastoplastic constitutive operator (taken from Bigoni and Petryk, 2002) embodying features typical of the behaviour of granular materials and capable of exhibiting flutter instability. An infinite body is considered made up of this material, homogeneously and quasi-statically deformed in two dimensions (plane strain or generalized plane stress). For this configuration a timeharmonic Green's function is found (in the way shown by Willis, 1991), which represents the first dynamic Green's function obtained for a nonsymmetric constitutive equation 2 .

\footnotetext{
${ }^{1}$ See Bigoni and Petryk (2002) for a discussion of this delicate assumption.

${ }^{2}$ A quasi-static Green's function for unsymmetric constitutive equation has been developed by Bertoldi et al. (2005), but this is unsuitable for flutter analyses, since this instability is essentially dynamic and thus remains unrevealed under the quasi-static assumption. In addition, Bertoldi et al. (2005) also derive boundary integral equations under the unsymmetric constitutive assumption, which are shown to possess certain typical features although not directly connected to the present discussion.
} 
The Green's function is employed to form a pulsating dipole (two equal and opposite forces having a magnitude varying sinusoidally with time) to be used as a dynamic perturbation revealing effects of flutter.

Results demonstrate the following features of flutter instability that may ${ }^{3}$ also occur in a material for which the tangent constitutive operator is positive definite (so that negative second-order work and shear bands are excluded at the considered stress level).

- Differently from shear bands, becoming already evident when the boundary of the region of ellipticity is approached from its interior (Bigoni and Capuani, 2002; 2005), flutter instability remains undetected while the eigenvalues of the acoustic tensor lie in the real range, appearing only after two real eigenvalues have coalesced and then become a complex conjugate pair;

- flutter instability corresponds to a disturbance blowing-up in space from the perturbing dipole and self-organizing along well-defined plane waves.

- the normals to the above plane waves lie within the fan of directions corresponding to flutter and have been found to have an inclination remarkably different from that corresponding to shear bands, occurring later in the hardening process.

It should be noted that the blow-up found in our solution will occur rapidly and nonlinearities neglected in our analysis (such as for instance the possibility of elastic unloading and plastic reloading) may soon become important, possibly changing the overall mechanical response. Equally significant is the fact that the rate of growth increases with the frequency that is adopted. The governing equations of motion thus represent a problem that is dynamically ill-posed in the general transient case, unless the tangent moduli in fact display a frequency-dependence, such that the flutter effect reduces as frequency increasest However, our results suggest that flutter instability should induce a layering in an initially homogeneous material, inducing a localization of strain in a form somehow similar - though possibly occurring much earlier in a hardening process - to that pertaining to shear bands occurring in a dynamical context (Bigoni and Capuani, 2005). Our hope is that this feature revealed by our results has now been made accessible to experimental investigation.

\section{$1.1 \quad$ Notation}

A standard, intrinsic notation is used throughout the paper (as for instance in Bigoni and Loret, 1999 and Bigoni, 2000), where vectors and second-order tensors are denoted

\footnotetext{
${ }^{3}$ More precisely, flutter instability has been shown by Bigoni and Loret (1999) to be unrelated to the occurrence of other instabilities such as loss of positive definiteness of second order work, loss of strong ellipticity and loss of ellipticity.

${ }^{4}$ Such a model was introduced by Bigoni and Willis (1994) in the context of a simple one-dimensional example.
} 
by bold (the latter capital) letters. The scalar product between arbitrary tensors $\boldsymbol{A}$ and $\boldsymbol{B}$ is denoted by

$$
\boldsymbol{A} \cdot \boldsymbol{B}=\operatorname{tr} \boldsymbol{A} \boldsymbol{B}^{T}=\boldsymbol{I} \cdot \boldsymbol{A} \boldsymbol{B}^{T},
$$

where the usual symbols denoting the identity, the transpose, and the trace operator have been employed. In addition to the usual tensorial product between (vectors and) second-order tensors $\boldsymbol{A}$ and $\boldsymbol{B}$

$$
(\boldsymbol{A} \otimes \boldsymbol{B})[\boldsymbol{C}]=(\boldsymbol{B} \cdot \boldsymbol{C}) \boldsymbol{A},
$$

for every $\boldsymbol{C}$, we will make use of the two tensorial products

$$
(\boldsymbol{A} \underline{\otimes} \boldsymbol{B})[\boldsymbol{C}]=\boldsymbol{A} \frac{\boldsymbol{C}+\boldsymbol{C}^{T}}{2} \boldsymbol{B}^{T}, \quad(\boldsymbol{A} \otimes \boldsymbol{B})[\boldsymbol{C}]=\boldsymbol{A} \boldsymbol{C} \boldsymbol{B}^{T}
$$

so that $\boldsymbol{I} \bar{\otimes} \boldsymbol{I}$ and $\boldsymbol{I} \otimes \boldsymbol{I}$ become the symmetrizing and the identity fourth-order tensors, respectively.

\section{A simple constitutive model evidencing flutter in- stability}

We refer here to the model proposed by Bigoni and Petryk (2002) as a large strain version of that proposed by Bigoni and Loret (1999) [see also Bigoni (1995) and Bigoni and Zaccaria (1994)]. In particular, an objective symmetric flux, namely, the Oldroyd derivative of the Kirchhoff stress

$$
\stackrel{\circ}{\boldsymbol{K}}=\dot{\boldsymbol{K}}-\boldsymbol{L} \boldsymbol{K}-\boldsymbol{K} \boldsymbol{L}^{T}
$$

(where a dot over a symbol denotes material time derivative, $\boldsymbol{L}=\dot{\boldsymbol{F}} \boldsymbol{F}^{-1}$ is the spatial velocity gradient and $\boldsymbol{F}$ the deformation gradient) is related to the Eulerian strain rate

$$
\boldsymbol{D}=\frac{1}{2}\left(\boldsymbol{L}+\boldsymbol{L}^{T}\right)
$$

through the piecewise-linear elastoplastic constitutive equation

$$
\stackrel{\circ}{\boldsymbol{K}}= \begin{cases}\mathbb{E}[\boldsymbol{D}]-\frac{1}{H}\langle\boldsymbol{Q} \cdot \mathbb{E}[\boldsymbol{D}]\rangle \mathbb{E}[\boldsymbol{P}] & \text { if } f(\boldsymbol{K}, \mathcal{K})=0, \\ \mathbb{E}[\boldsymbol{D}] & \text { if } f(\boldsymbol{K}, \mathcal{K})<0,\end{cases}
$$

where the symbol $\langle\cdot\rangle$ denotes the Macaulay brackets operator (defined for every scalar $\alpha$ as $\langle\alpha\rangle=(\alpha+|\alpha|) / 2), \mathbb{E}$ is the elastic fourth-order tensor, $f$ is the yield function in stress space depending on a collection $\mathcal{K}$ of internal variables (of arbitrary scalar or tensorial 
nature); moreover, $\boldsymbol{P}$ and $\boldsymbol{Q}$ are the normals to the plastic potential and yield surface, respectively, and the plastic modulus $H$ is related to the hardening modulus $h$ through

$$
H=h+\boldsymbol{Q} \cdot \mathbb{E}[\boldsymbol{P}]
$$

In the present article, we will refer to the loading branch of eqn. (마), which is

$$
\dot{\boldsymbol{K}}=\mathbb{E}[\boldsymbol{L}]+\boldsymbol{L} \boldsymbol{K}+\boldsymbol{K} \boldsymbol{L}^{T}-\frac{1}{H}\left(\mathbb{E}[\boldsymbol{P}] \otimes \mathbb{E}^{T}[\boldsymbol{Q}]\right)[\boldsymbol{L}]
$$

where we have used the minor symmetries of $\mathbb{E}$. Finally, introducing the first PiolaKirchhoff stress

$$
\boldsymbol{S}=\boldsymbol{K} \boldsymbol{F}^{-T}
$$

eqn. (8) can be rewritten as

$$
\dot{\boldsymbol{S}}=\mathbb{C}[\dot{\boldsymbol{F}}]
$$

where

$$
\begin{aligned}
\mathbb{C}=\left(\boldsymbol{I} \otimes \boldsymbol{F}^{-1}\right) \mathbb{E}\left(\boldsymbol{I} \otimes \boldsymbol{F}^{-T}\right) & +\boldsymbol{I} \otimes \boldsymbol{F}^{-1} \boldsymbol{S} \\
& -\frac{1}{H}\left(\boldsymbol{I} \otimes \boldsymbol{F}^{-1}\right)(\mathbb{E}[\boldsymbol{P}] \otimes \mathbb{E}[\boldsymbol{Q}])\left(\boldsymbol{I} \otimes \boldsymbol{F}^{-T}\right) .
\end{aligned}
$$

Note that the tangent constitutive operator $\mathbb{C}$, eqn. (11), possesses neither the minor nor the major symmetry, the latter except in the associative case, $\boldsymbol{Q}=\boldsymbol{P}$.

\subsection{Anisotropic elasticity}

Following Bigoni and Loret (1999) an anisotropic elastic law is assumed in the form

$$
\mathbb{E}=\lambda \boldsymbol{B} \otimes \boldsymbol{B}+2 \mu \boldsymbol{B} \underline{\bar{\otimes}} \boldsymbol{B},
$$

where $\lambda$ and $\mu$ are material constants subject to the restrictions $\mu>0,3 \lambda+2 \mu>0$, and $\boldsymbol{B}$ is a symmetric, positive definite second-order tensor, selected in the format

$$
\boldsymbol{B}=b_{1} \boldsymbol{b} \otimes \boldsymbol{b}+b_{2}(\boldsymbol{I}-\boldsymbol{b} \otimes \boldsymbol{b}),
$$

where $b_{1}$ and $b_{2}$ are the eigenvalues of $\boldsymbol{B}$, while the line spanned by the unit vector $\boldsymbol{b}$ and the plane perpendicular to it are the corresponding eigenspaces. Moreover, the material constants $b_{1}$ and $b_{2}$ are assumed to depend on a single angular parameter $\hat{b}$, restricted to the range $] 0^{\circ}, 90^{\circ}$ [ to meet the positive definiteness requirement of $\boldsymbol{B}$,

$$
b_{1}=\sqrt{3} \cos \hat{b}, \quad b_{2}=\sqrt{\frac{3}{2}} \sin \hat{b}
$$

so that the isotropic behaviour is recovered when $b_{1}=b_{2}=1$, or $\hat{b} \approx 54.74^{\circ}$. 


\subsection{The acoustic tensor}

The acoustic tensor $\boldsymbol{A}^{e p}(\boldsymbol{n})$ associated with the tangent constitutive operator $\mathbb{C}$ and the mass density $\rho$ is defined by

$$
\boldsymbol{A}^{e p}(\boldsymbol{n}) \boldsymbol{g}=\frac{1}{\rho} \mathbb{C}[\boldsymbol{g} \otimes \boldsymbol{n}] \boldsymbol{n},
$$

where $\boldsymbol{n}$ and $\boldsymbol{g}$ are the direction and amplitude of the propagating wave, respectively. Therefore, the acoustic tensor corresponding to $\mathbb{C}$ in eqn. (11) is

$$
\boldsymbol{A}^{e p}(\boldsymbol{n})=\boldsymbol{A}^{e}(\boldsymbol{n})-\frac{1}{\rho H}\left(\mathbb{E}[\boldsymbol{P}] \boldsymbol{F}^{-T} \boldsymbol{n} \otimes \mathbb{E}[\boldsymbol{Q}] \boldsymbol{F}^{-T} \boldsymbol{n}\right),
$$

where $\boldsymbol{A}^{e}(\boldsymbol{n})$ is the elastic acoustic tensor, defined as

$$
\begin{aligned}
& \boldsymbol{A}^{e}(\boldsymbol{n})=\frac{\lambda+\mu}{\rho}\left(\boldsymbol{B} \boldsymbol{F}^{-T} \boldsymbol{n}\right) \otimes\left(\boldsymbol{B F}^{-T} \boldsymbol{n}\right) \\
& +\frac{\mu}{\rho}\left[\left(\boldsymbol{F}^{-T} \boldsymbol{n}\right) \cdot\left(\boldsymbol{B} \boldsymbol{F}^{-T} \boldsymbol{n}\right)\right] \boldsymbol{B}+\frac{1}{\rho}\left[\boldsymbol{n} \cdot\left(\boldsymbol{F}^{-1} \boldsymbol{S n}\right)\right] \boldsymbol{I} .
\end{aligned}
$$

Since $\mathbb{C}$ does not have the major symmetry, the acoustic tensor (16)-(17) is also not symmetric.

\subsection{Examples of flutter instability for plane problems}

The current configuration is assumed as reference, so that $\boldsymbol{F}=\boldsymbol{I}$ and $\boldsymbol{S}=\boldsymbol{K}=\boldsymbol{T}$, where $\boldsymbol{T}$ denotes the Cauchy stress. The plane problem is considered in which vector $\boldsymbol{b}$ and the propagation direction $\boldsymbol{n}$ lie in the plane spanned by $\boldsymbol{k}_{1}$ and $\boldsymbol{k}_{2}$, two unit eigenvectors of $\boldsymbol{K}=\boldsymbol{T}$. Assuming the Drucker-Prager yield criterion, tensors $\boldsymbol{P}$ and $\boldsymbol{Q}$ take the form

$$
\boldsymbol{P}=\cos \chi \frac{\operatorname{dev} \boldsymbol{T}}{|\operatorname{dev} \boldsymbol{T}|}+\frac{\sin \chi}{\sqrt{3}} \boldsymbol{I}, \quad \boldsymbol{Q}=\cos \psi \frac{\operatorname{dev} \boldsymbol{T}}{|\operatorname{dev} \boldsymbol{T}|}+\frac{\sin \psi}{\sqrt{3}} \boldsymbol{I},
$$

respectively, where $\operatorname{dev} \boldsymbol{T}=\boldsymbol{T}-\operatorname{tr} \boldsymbol{T} / 3$ and the angular parameters $\chi$ and $\psi$ describe respectively the dilatancy and the pressure-sensitivity of the material.

In the reference system $\left\{\boldsymbol{n}, \boldsymbol{s}, \boldsymbol{k}_{3}\right\}$, where $\boldsymbol{s}=\boldsymbol{k}_{3} \times \boldsymbol{n}$, the acoustic tensor $\boldsymbol{A}^{e p}(\boldsymbol{n})$ becomes

$$
\left(\begin{array}{ccc}
A_{n n}^{e}-\frac{1}{\rho H}(\boldsymbol{n} \cdot \boldsymbol{q})(\boldsymbol{n} \cdot \boldsymbol{p}) & A_{n s}^{e}-\frac{1}{\rho H}(\boldsymbol{n} \cdot \boldsymbol{q})(\boldsymbol{s} \cdot \boldsymbol{p}) & 0 \\
A_{n s}^{e}-\frac{1}{\rho H}(\boldsymbol{s} \cdot \boldsymbol{q})(\boldsymbol{n} \cdot \boldsymbol{p}) & A_{s s}^{e}-\frac{1}{\rho H}(\boldsymbol{s} \cdot \boldsymbol{q})(\boldsymbol{s} \cdot \boldsymbol{p}) & 0 \\
0 & 0 & \frac{\mu b_{2}(\boldsymbol{n} \cdot \boldsymbol{B} \boldsymbol{n})+\boldsymbol{n} \cdot \boldsymbol{T n}}{\rho}
\end{array}\right)
$$


where

$$
\begin{aligned}
\boldsymbol{q} & \equiv \mathbb{E}[\boldsymbol{Q}] \boldsymbol{n}=\lambda(\boldsymbol{B} \cdot \boldsymbol{Q}) \boldsymbol{B} \boldsymbol{n}+2 \mu \boldsymbol{B} \boldsymbol{Q} \boldsymbol{B} \boldsymbol{n}, \\
\boldsymbol{p} & \equiv \mathbb{E}[\boldsymbol{P}] \boldsymbol{n}=\lambda(\boldsymbol{B} \cdot \boldsymbol{P}) \boldsymbol{B} \boldsymbol{n}+2 \mu \boldsymbol{B} \boldsymbol{P} \boldsymbol{B} \boldsymbol{n},
\end{aligned}
$$

and $A_{n n}^{e}, A_{s s}^{e}, A_{n s}^{e}$ are the in-plane components of the elastic acoustic tensor $\boldsymbol{A}^{e}(\boldsymbol{n})$, namely

$$
\begin{aligned}
& A_{n n}^{e}=\frac{\lambda+2 \mu}{\rho}(\boldsymbol{n} \cdot \boldsymbol{B} \boldsymbol{n})^{2}+\frac{1}{\rho} \boldsymbol{n} \cdot \boldsymbol{T} \boldsymbol{n}, \\
& A_{s s}^{e}=\frac{\lambda+\mu}{\rho}(\boldsymbol{s} \cdot \boldsymbol{B} \boldsymbol{n})^{2}+\frac{\mu}{\rho}(\boldsymbol{n} \cdot \boldsymbol{B} \boldsymbol{n})(\boldsymbol{s} \cdot \boldsymbol{B} \boldsymbol{s})+\frac{1}{\rho} \boldsymbol{n} \cdot \boldsymbol{T} \boldsymbol{n}, \\
& A_{n s}^{e}=\frac{\lambda+2 \mu}{\rho}(\boldsymbol{n} \cdot \boldsymbol{B} \boldsymbol{n})(\boldsymbol{s} \cdot \boldsymbol{B} \boldsymbol{n}) .
\end{aligned}
$$

Note that the out-of-plane eigenvalue $A_{33}^{e p}$ in eqn. (19) corresponds to a wave with outof-plane amplitude $\left(\boldsymbol{g}\right.$ proportional to $\left.\boldsymbol{k}_{3}\right)$ and is assumed to remain strictly positive.

From matrix (19), we get the sum and the product of the two in-plane eigenvalues (squares of the acceleration waves propagation velocities) $c_{1}^{2}$ and $c_{2}^{2}$ corresponding to waves with in-plane amplitude $\left(\boldsymbol{g}\right.$ lying in the plane spanned by $\boldsymbol{k}_{1}$ and $\left.\boldsymbol{k}_{2}\right)$,

$$
\begin{aligned}
& c_{1}^{2}+c_{2}^{2}=A_{n n}^{e}+A_{s s}^{e}-\frac{1}{\rho H}\left(f_{1}-f_{2}\right), \\
& c_{1}^{2} c_{2}^{2}=A_{n n}^{e} A_{s s}^{e}-\left(A_{n s}^{e}\right)^{2}+\frac{1}{\rho H}\left(A_{n s}^{e} f_{3}-A_{s s}^{e} f_{1}+A_{n n}^{e} f_{2}\right),
\end{aligned}
$$

where

$$
\begin{aligned}
& f_{1}=(\boldsymbol{n} \cdot \boldsymbol{q})(\boldsymbol{n} \cdot \boldsymbol{p}), \quad f_{2}=-(\boldsymbol{s} \cdot \boldsymbol{q})(\boldsymbol{s} \cdot \boldsymbol{p}), \\
& f_{3}=(\boldsymbol{n} \cdot \boldsymbol{q})(\boldsymbol{s} \cdot \boldsymbol{p})+(\boldsymbol{s} \cdot \boldsymbol{q})(\boldsymbol{n} \cdot \boldsymbol{p}) .
\end{aligned}
$$

A necessary and sufficient condition for the existence of complex conjugate eigenvalues $a_{1}^{e p}$ and $a_{2}^{e p}$ is represented by the simultaneous fulfillment of the following three conditions (Bigoni and Loret, 1999)

$$
\begin{aligned}
& f_{4}=\left(A_{n n}^{e}-A_{s s}^{e}\right)^{2}\left[\left(f_{1}+f_{2}+2 e f_{3}\right)^{2}-\left(1+4 e^{2}\right)\left(f_{1}-f_{2}\right)^{2}\right]>0, \\
& f_{5}=\left(A_{n n}^{e}-A_{s s}^{e}\right)\left(f_{1}+f_{2}+2 e f_{3}\right)>0, \\
& \frac{f_{5}-\sqrt{f_{4}}}{\left(A_{n n}^{e}-A_{s s}^{e}\right)^{2}+4\left(A_{n s}^{e}\right)^{2}}<\rho^{2} H<\frac{f_{5}+\sqrt{f_{4}}}{\left(A_{n n}^{e}-A_{s s}^{e}\right)^{2}+4\left(A_{n s}^{e}\right)^{2}},
\end{aligned}
$$

where

$$
e=\frac{A_{n s}^{e}}{A_{n n}^{e}-A_{s s}^{e}} .
$$


With reference to Fig. 1, let $\theta_{\sigma}$ and $\theta_{n}$ be the angles of inclination of the direction of elastic anisotropy $\boldsymbol{b}$ and wave propagation normal $\boldsymbol{n}$ with respect to the stress principal axis $\boldsymbol{k}_{1}$.

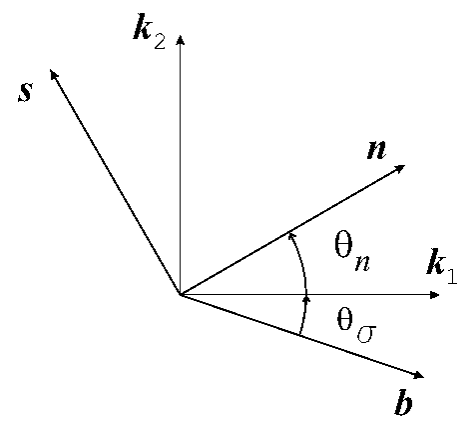

Figure 1: Principal stress axes $\boldsymbol{k}_{1}$ and $\boldsymbol{k}_{2}$, axis of elastic symmetry $\boldsymbol{b}$ and propagation direction $\boldsymbol{n}$, singled out by angles $\theta_{\sigma}$ and $\theta_{n}$, respectively.

Dividing all quantities having the dimension of a stress in eqns. (19)-(24) by $\mu$, the parameters on which the condition of flutter depends are:

- Elastic parameters: $\lambda / \mu$, strength of anisotropy $\hat{b}$, and orientation of the axis of elastic symmetry with respect to the principal stress axis $\boldsymbol{k}_{1}$, namely, $\theta_{\sigma}$.

- Plastic parameters: plastic modulus $H / \mu$, pressure sensitivity $\psi$, and dilatancy $\chi$ parameters.

- Principal normalized deviatoric stress values: $\operatorname{dev} T_{1} /|\operatorname{dev} \boldsymbol{T}|, \operatorname{dev} T_{2} /|\operatorname{dev} \boldsymbol{T}|, \operatorname{dev} T_{3} /|\operatorname{dev} \boldsymbol{T}|$. However, these are not independent, so that given the form (18) of $\boldsymbol{P}$ and $\boldsymbol{Q}$, flutter depends on the angle

$$
\theta_{L}=\operatorname{sgn}\left(\frac{\operatorname{dev} T_{1}}{|\operatorname{dev} \boldsymbol{T}|}+2 \frac{\operatorname{dev} T_{2}}{|\operatorname{dev} \boldsymbol{T}|}\right) \cos ^{-1}\left(\sqrt{\frac{3}{2}} \frac{\operatorname{dev} T_{1}}{|\operatorname{dev} \boldsymbol{T}|}\right)
$$

in the deviatoric plane, which is a 'modified Lode angle', defined for $\theta_{L} \in[-\pi, \pi]$ and in which $\operatorname{sgn}(0)=1$.

It is possible to study flutter for all the propagation directions $\boldsymbol{n}$ while varying the plastic modulus $H / \mu$ and all remaining parameters in the above list are kept fixed, by use of inequalities (24). Therefore, the ranges in which flutter occurs can be plotted in the plane $H / \mu$ versus $\theta_{n}$. Restricting the analysis to the infinitesimal theory, where the flux (4) is identified with $\dot{\boldsymbol{T}}$, analyses have been performed for simplicity with different values of the modified Lode parameter $\theta_{L}=\left\{60^{\circ}, 30^{\circ}, 0^{\circ},-30^{\circ},-60^{\circ}\right\}$, as indicated in Fig. 2, 


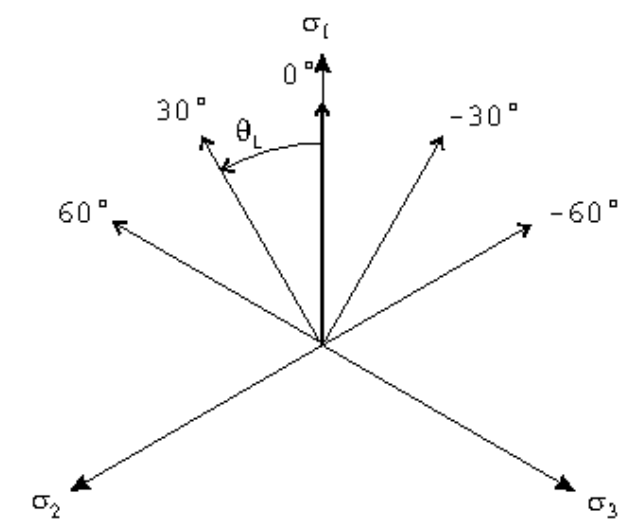

Figure 2: Stress directions in the deviatoric plane, defined by the modified Lode angle (26), considered for flutter analysis.

Results are reported in Figs. 3 and 4, the latter giving more detail for four of the cases reported in the former figure. Different stress paths defined by the values of the modified Lode angle (26) reported in Fig. 2 are considered for different anisotropy inclination $\theta_{\sigma}$ in Fig. 3 at given values of $\psi=30^{\circ}$ and $\chi=0^{\circ}$. In the graphs the closed contours denote regions where flutter occurs in the plane defined by the normalized critical plastic modulus $H / \mu$ and the inclination of propagation direction $\theta_{n}$.

Four details of Fig. 3 are reported in Fig. 4, where $\lambda / \mu=1, \hat{b}=80^{\circ}, \psi=30^{\circ}$, and $\chi=0^{\circ}$, as in Fig. 3. The six regions in Fig. 4 correspond to the four cases $\theta_{L}=0^{\circ}$ and $\theta_{\sigma}=15^{\circ}($ Case 1$), \theta_{L}=\theta_{\sigma}=30^{\circ}($ Case 2$), \theta_{L}=0$ and $\theta_{\sigma}=45^{\circ}$ (Case 3), and $\theta_{L}=0$ and $\theta_{\sigma}=60^{\circ}$ (Case 4 ).

With reference to the Cases 1,2,3 and 4, detailed in Fig. 4, we note that the critical values of plastic modulus for loss of positive definiteness of the constitutive operator $H_{c r}^{P D}$ and for loss of ellipticity $H_{c r}^{E}$ permitting shear bands with normal inclined at $\theta_{n E}$ are 5 :

Case 1: $H_{c r}^{P D} / \mu=0.42, \quad H_{c r}^{E} / \mu=0.19, \quad \theta_{n E}=-28.0^{\circ}$,

Case 2: $\quad H_{c r}^{P D} / \mu=1.22, \quad H_{c r}^{E} / \mu=0.18, \quad \theta_{n E}=-16.4^{\circ}$,

Case 3: $\quad H_{c r}^{P D} / \mu=1.03, \quad H_{c r}^{E} / \mu=0.74, \quad \theta_{n E}=-32.0^{\circ}$,

Case 4: $H_{c r}^{P D} / \mu=1.84, \quad H_{c r}^{E} / \mu=1.57, \quad \theta_{n E}=-33.9^{\circ}$,

so that in all cases flutter may initiate when the constitutive operator is positive definite

\footnotetext{
${ }^{5}$ Note that with 'ellipticity loss' we mean here the condition pertinent to the underlying quasi-static deformation. Moreover, due to anisotropy, only one shear band is found as first noticed by Bigoni et al. (2000).
} 
(therefore at an early stage of a deformation process) and may extend in a region possibly involving loss of ellipticity. Note that thresholds (27) have been graphically represented in Fig, 4, where light grey regions correspond to regions where flutter may occur with the constitutive operator still positive definite, while in the dark grey regions ellipticity is lost (horizontal lines marking ellipticity loss are denoted with 'E (case i)', where $\mathrm{i}=$ $1, . .4$ stands for the number of the relevant Case). In the same figure, three black spots and a white spot (referred to Case 2) indicate the inclinations of shear bands at first loss of ellipticity. Note that the small flutter regions of Cases 3 and 4 are beyond the positive definiteness threshold, but still in the elliptic region. It may be important to remark that

the initial inclinations of propagation normals for flutter and shear bands are unrelated and remarkably different.

From the above analysis it can be deduced that the constitutive model allows one to approach flutter starting from a well-behaved state. Moreover, it may be interesting to note from Fig. 4 that there are overlapping regions corresponding to different stress states (Cases 1 and 2). In these zones the flutter may have identical characteristics even if the stress state is different.

\subsection{Spectral analysis of the acoustic tensor}

The spectral analysis of the acoustic tensor is instrumental to the development of the Green's function that will be presented in the next Section. The analysis is restricted to the in-plane components of the acoustic tensor $\boldsymbol{A}^{e p}$

$$
\boldsymbol{A}=A_{11}^{e p}\left(\boldsymbol{k}_{1} \otimes \boldsymbol{k}_{1}\right)+A_{12}^{e p}\left(\boldsymbol{k}_{1} \otimes \boldsymbol{k}_{2}\right)+A_{21}^{e p}\left(\boldsymbol{k}_{2} \otimes \boldsymbol{k}_{1}\right)+A_{22}^{e p}\left(\boldsymbol{k}_{2} \otimes \boldsymbol{k}_{2}\right),
$$

represented for later convenience in the principal stress basis $\boldsymbol{k}_{1}, \boldsymbol{k}_{2}$. The inverse of (28) can be written as

$$
\begin{aligned}
& \boldsymbol{A}^{-1}=\frac{1}{A_{11}^{e p} A_{22}^{e p}-A_{12}^{e p} A_{21}^{e p}}\left[A_{22}^{e p}\left(\boldsymbol{k}_{1} \otimes \boldsymbol{k}_{1}\right)-A_{12}^{e p}\left(\boldsymbol{k}_{1} \otimes \boldsymbol{k}_{2}\right)\right. \\
&\left.-A_{21}^{e p}\left(\boldsymbol{k}_{2} \otimes \boldsymbol{k}_{1}\right)+A_{11}^{e p}\left(\boldsymbol{k}_{2} \otimes \boldsymbol{k}_{2}\right)\right] .
\end{aligned}
$$

Now, the eigenvalues of the acoustic tensor (28) can be written in the form

$$
\left.\begin{array}{l}
c_{1}^{2} \\
c_{2}^{2}
\end{array}\right\}=\frac{A_{11}^{e p}+A_{22}^{e p} \pm \Delta}{2}, \quad \Delta=\sqrt{\left(A_{11}^{e p}-A_{22}^{e p}\right)^{2}+4 A_{12}^{e p} A_{21}^{e p}},
$$

so that assuming non-defectiveness, the spectral representations of $\boldsymbol{A}$ and $\boldsymbol{A}^{-1}$ are

$$
\boldsymbol{A}=c_{1}^{2}\left(\boldsymbol{v}_{1} \otimes \boldsymbol{w}_{1}\right)+c_{2}^{2}\left(\boldsymbol{v}_{2} \otimes \boldsymbol{w}_{2}\right)
$$




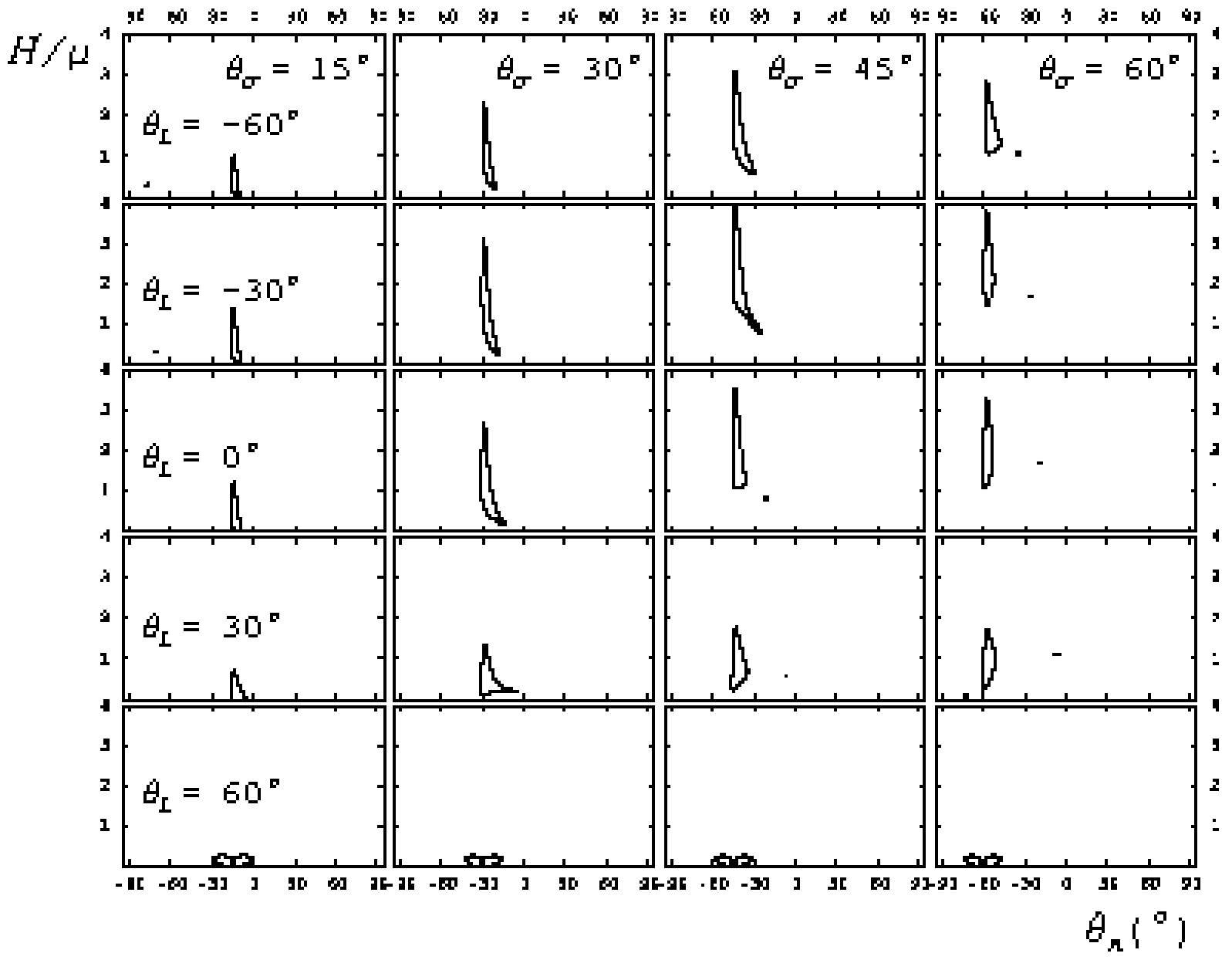

Figure 3: Regions of flutter instability (occurring for internal points) in the $H / \mu$ vs. $\theta_{n}$ plane, for the stress paths shown in Fig. 2 at various anisotropy inclinations $\theta_{\sigma}$. The following values of material parameters have been considered: $\lambda / \mu=1, \hat{b}=80^{\circ}, \psi=30^{\circ}$, and $\chi=0^{\circ}$.

and, assuming ${ }^{6} c_{1}^{2} \neq 0$ and $c_{2}^{2} \neq 0$,

$$
\boldsymbol{A}^{-1}=\frac{1}{c_{1}^{2}}\left(\boldsymbol{v}_{1} \otimes \boldsymbol{w}_{1}\right)+\frac{1}{c_{2}^{2}}\left(\boldsymbol{v}_{2} \otimes \boldsymbol{w}_{2}\right)
$$

where $\left\{\boldsymbol{v}_{1}, \boldsymbol{v}_{2}\right\}$ and $\left\{\boldsymbol{w}_{1}, \boldsymbol{w}_{2}\right\}$ are dual bases, thus satisfying $\boldsymbol{v}_{i} \cdot \boldsymbol{w}_{j}=\delta_{i j}(i, j=1,2)$,

${ }^{6}$ For $\Delta \rightarrow 0$ (coalescence of the eigenvalues), the tensor $\boldsymbol{A}$ becomes defective (except for the trivial case where $\boldsymbol{A}$ is isotropic) and each term in the spectral representation of $\boldsymbol{A}$, and also of $\boldsymbol{A}^{-1}$, blows up but $\boldsymbol{A}^{-1}$ continues to exist and to be defined correctly. Indeed a substitution of eqns. (30) and (33) or (34) into eqn. (32) leads to eqn. (29). 


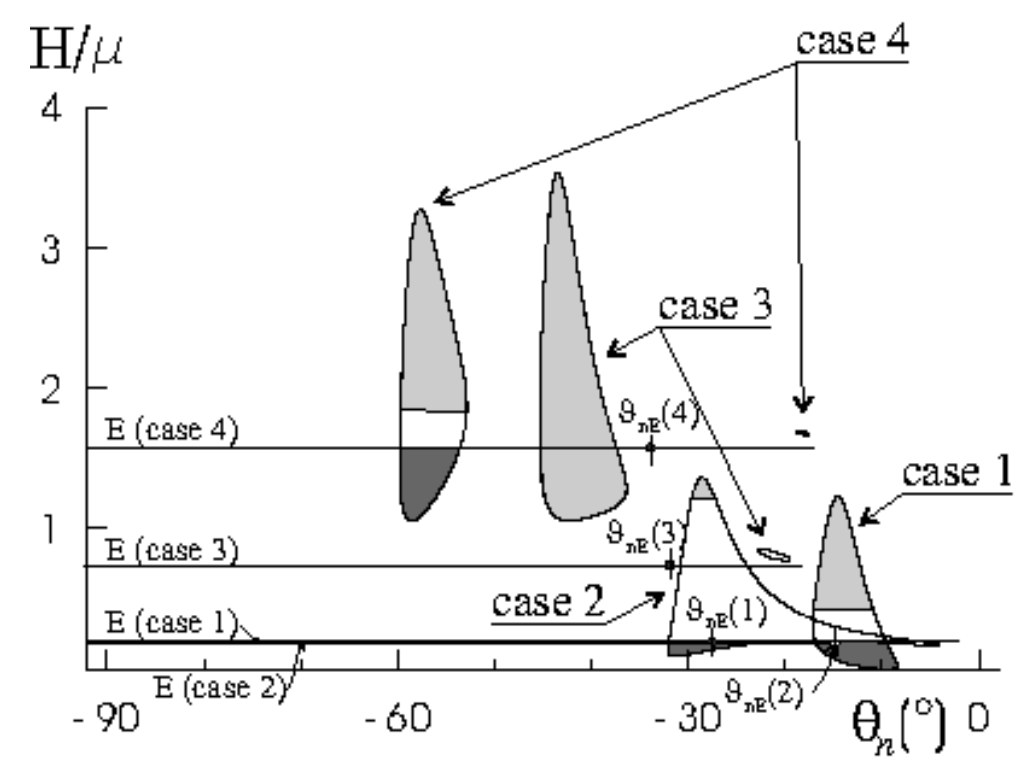

Figure 4: Regions of flutter instability (occurring for internal points) in the $H / \mu$ vs. $\theta_{n}$ plane, for $\lambda / \mu=1, \hat{b}=80^{\circ}, \psi=30^{\circ}$, and $\chi=0^{\circ}$. Case 1: $\theta_{L}=0^{\circ}$ and $\theta_{\sigma}=15^{\circ}$. Case 2: $\theta_{L}=30^{\circ}$ and $\theta_{\sigma}=30^{\circ}$. Case 3: as in case 1 , but $\theta_{\sigma}=45^{\circ}$. Case 4: as in case 1 , but $\theta_{\sigma}=60^{\circ}$. The regions of positive definiteness of the constitutive operator are marked in light grey, while (E) denotes loss of ellipticity into shear bands (regions shaded in dark grey) inclined at $\theta_{n E}(i)$, where $\mathrm{i}=1, . ., 4$ denotes the relevant Case.

composed of right, $\boldsymbol{v}_{i}$, and left, $\boldsymbol{w}_{i}$, eigenvectors. This basis is given by

$$
\begin{array}{ll}
\boldsymbol{v}_{1}=\boldsymbol{k}_{1}+\frac{\Delta-\left(A_{11}^{e p}-A_{22}^{e p}\right)}{2 A_{12}^{e p}} \boldsymbol{k}_{2}, & \boldsymbol{v}_{2}=\boldsymbol{k}_{1}+\frac{-\Delta-\left(A_{11}^{e p}-A_{22}^{e p}\right)}{2 A_{12}^{e p}} \boldsymbol{k}_{2}, \\
\boldsymbol{w}_{1}=\frac{\Delta+\left(A_{11}^{e p}-A_{22}^{e p}\right)}{2 \Delta} \boldsymbol{k}_{1}+\frac{A_{12}^{e p}}{\Delta} \boldsymbol{k}_{2}, & \boldsymbol{w}_{2}=\frac{\Delta-\left(A_{11}^{e p}-A_{22}^{e p}\right)}{2 \Delta} \boldsymbol{k}_{1}-\frac{A_{12}^{e p}}{\Delta} \boldsymbol{k}_{2},
\end{array}
$$

when $A_{12}^{e p} \neq 0$, or by

$$
\begin{array}{ll}
\boldsymbol{v}_{1}=\frac{\Delta+\left(A_{11}^{e p}-A_{22}^{e p}\right)}{2 A_{21}^{e p}} \boldsymbol{k}_{1}+\boldsymbol{k}_{2}, & \boldsymbol{v}_{2}=\frac{-\Delta+\left(A_{11}^{e p}-A_{22}^{e p}\right)}{2 A_{21}^{e p}} \boldsymbol{k}_{1}+\boldsymbol{k}_{2}, \\
\boldsymbol{w}_{1}=\frac{A_{21}^{e p}}{\Delta} \boldsymbol{k}_{1}+\frac{\Delta-\left(A_{11}^{e p}-A_{22}^{e p}\right)}{2 \Delta} \boldsymbol{k}_{2}, & \boldsymbol{w}_{2}=-\frac{A_{21}^{e p}}{\Delta} \boldsymbol{k}_{1}+\frac{\Delta+\left(A_{11}^{e p}-A_{22}^{e p}\right)}{2 \Delta} \boldsymbol{k}_{2},
\end{array}
$$

when $A_{21}^{e p} \neq 0$. The case $A_{21}^{e p}=A_{12}^{e p}=0$ is trivial. 


\section{The dynamic time-harmonic Green's function for general nonsymmetric constitutive equations}

An initial static homogeneous deformation of an infinite body is considered, satisfying equilibrium in terms of first Piola-Kirchhoff stress, namely,

$$
\operatorname{div} \boldsymbol{S}=\mathbf{0},
$$

and taken as the reference state in an updated Lagrangian formulation. A dynamic perturbation is superimposed upon this state, defined by an incremental displacement $\boldsymbol{u}$ satisfying the equations of incremental motion, written with reference to the constitutive equation (10) in which dotted symbols are to be interpreted now as incremental quantities rather than rates. Thus

$$
\mathbb{C}_{i j k l} u_{k, l j}+f_{i}=\rho u_{i, t t},
$$

where ${ }_{t}$ denotes material time derivative and $f_{i}$ and $\rho$ are the incremental body forces and the mass density, respectively.

Equations (36) look like ordinary elastodynamics, except that

$$
\begin{aligned}
& \mathbb{C}_{i j k l} \text { has neither the usual major } \mathbb{C}_{i j k l} \neq \mathbb{C}_{k l i j} \text { nor the minor } \mathbb{C}_{i j l k} \neq \mathbb{C}_{i j k l} \neq \\
& \mathbb{C}_{j i k l} \text { symmetries. }
\end{aligned}
$$

Note that tensor $\mathbb{C}_{i j k l}$ can be identified (and will be in the examples) with that provided by eqn. (11), but can also be thought completely arbitrary in the following. To investigate the properties of eqn. (36), outside and inside the flutter region we follow the Bigoni and Capuani $(2002 ; 2005)$ approach, based on the determination of the dynamic Green's function, sought for simplicity under the time-harmonic assumption

$$
u_{i}(\boldsymbol{x}, t)=\hat{u}_{i}(\boldsymbol{x}) e^{-i \omega t}, \quad f_{i}(\boldsymbol{x}, t)=\hat{f}_{i}(\boldsymbol{x}) e^{-i \omega t},
$$

where $\omega$ is the circular frequency and $t$ and $\boldsymbol{x}$ denote time and space variables, respectively, so that the time dependence can be removed from eqn. (36) and consequently

$$
\mathbb{C}_{i j k l} \hat{u}_{k, l j}+\rho \omega^{2} \hat{u}_{i}+\hat{f}_{i}=0 .
$$

The Green's tensor $G_{i p}(\boldsymbol{x})$ is obtained by solving eqn. (38) under the hypothesis $\hat{f}_{i}=$ $\delta_{i p} \delta(\boldsymbol{x})$, with $\delta(\boldsymbol{x})$ denoting the Dirac delta. We obtain

$$
\mathbb{C}_{i j k l} G_{k q, l j}(\boldsymbol{x})+\rho \omega^{2} G_{i q}(\boldsymbol{x})+\delta_{i q} \delta(\boldsymbol{x})=0 .
$$

In order to approach the flutter condition, we exploit the analysis of the acoustic tensor developed for the planar problem in Section 2.3, considering an infinite medium subject to plane strain (or generalized plane stress conditions), in which only four relevant components of the Green's function appear

$$
G_{i q}=G_{i q}\left(x_{1}, x_{2}\right), \quad i, q=\{1,2\},
$$

and depend only on the two coordinates $x_{1}$ and $x_{2}$. 


\subsection{Radon transform}

The Green's function is determined employing a Radon transform technique [the alternative approach employed by Bigoni and Capuani (2005) and based on a plane wave expansion is presented for completeness in Appendix A]. The Radon transform of a generic function $f(\boldsymbol{x}), \boldsymbol{x} \in \mathbf{R}^{2}$ is defined as

$$
\mathcal{R}[f(\boldsymbol{x})]=\hat{f}(p, \boldsymbol{n})=\int_{\mathbf{R}^{2}} f(\boldsymbol{x}) \delta(p-\boldsymbol{n} \cdot \boldsymbol{x}) d \boldsymbol{x}, \quad p \in \mathbf{R}, \boldsymbol{n} \in \mathbf{R}^{2}
$$

with the inverse

$$
f(\boldsymbol{x})=\frac{1}{4 \pi^{2}} \int_{|\boldsymbol{n}|=1} f_{-\infty}^{+\infty} \frac{\hat{f}^{\prime}(p, \boldsymbol{n})}{(\boldsymbol{n} \cdot \boldsymbol{x}-p)} d p d s,
$$

where a prime denotes partial differentiation in the following way

$$
\hat{f}^{\prime}(p, \boldsymbol{n})=\frac{\partial \hat{f}(p, \boldsymbol{n})}{\partial p} .
$$

In addition to the linearity, we will make use of the following properties of the Radon transform:

- derivative transforms

$$
\mathcal{R}\left[f_{, j}(\boldsymbol{x})\right]=n_{j} \hat{f}^{\prime}(p, \boldsymbol{n}), \quad \mathcal{R}\left[f_{, l j}(\boldsymbol{x})\right]=n_{l} n_{j} \hat{f}^{\prime \prime}(p, \boldsymbol{n}),
$$

- transform of the two-dimensional Dirac delta function

$$
\mathcal{R}[\delta(\boldsymbol{x})]=\delta(p)
$$

The Radon transform of eqn. (39) is therefore

$$
\mathbb{C}_{i j k l} n_{l} n_{j} \hat{G}_{k q}^{\prime \prime}(p, \boldsymbol{n})+\rho \omega^{2} \hat{G}_{i q}(p, \boldsymbol{n})+\delta_{i q} \delta(p)=0,
$$

where

$$
\hat{G}_{k q}^{\prime \prime}(p, \boldsymbol{n})=\frac{\partial^{2}}{\partial p^{2}} \hat{G}_{k q}(p, \boldsymbol{n})
$$

Eqn. (46) can be rewritten in tensorial form as

$$
\boldsymbol{A}(\boldsymbol{n}) \hat{\boldsymbol{G}}^{\prime \prime}(p, \boldsymbol{n})+\omega^{2} \hat{\boldsymbol{G}}(p, \boldsymbol{n})+\frac{\delta(p)}{\rho} \boldsymbol{I}=\mathbf{0} .
$$

Let us assume that $\boldsymbol{A}(\boldsymbol{n})$ has two non-null and distinct eigenvalues $c_{N}^{2}$ and corresponding left and right eigenvectors $\boldsymbol{w}_{N}, \boldsymbol{v}_{N},(N=1,2)$, which can be used as dual basis 
vectors, therefore satisfying $\boldsymbol{v}_{N} \cdot \boldsymbol{w}_{M}=\delta_{N M},(N, M=1,2)$. Employing the spectral representations of $\boldsymbol{A}(\boldsymbol{n})$ and $\boldsymbol{I}$

$$
\boldsymbol{A}(\boldsymbol{n})=\sum_{N=1}^{2} c_{N}^{2} \boldsymbol{v}_{N} \otimes \boldsymbol{w}_{N}, \quad \boldsymbol{I}=\sum_{N=1}^{2} \boldsymbol{v}_{N} \otimes \boldsymbol{w}_{N}
$$

in eqns. (48) and representing the transformed Green's function as

$$
\hat{\boldsymbol{G}}(p, \boldsymbol{n})=\sum_{N=1}^{2} \phi_{N}(p, \boldsymbol{n}) \boldsymbol{v}_{N} \otimes \boldsymbol{w}_{N},
$$

where $\phi_{N}$ is a (for the moment unknown) function of $p$ and $\boldsymbol{n}$, we get

$$
\sum_{N=1}^{2}\left[c_{N}^{2} \phi_{N}^{\prime \prime}+\omega^{2} \phi_{N}+\frac{\delta(p)}{\rho}\right] \boldsymbol{v}_{N} \otimes \boldsymbol{w}_{N}=\mathbf{0}
$$

which is equivalent to the following uncoupled system of two equations,

$$
\phi_{N}^{\prime \prime}+k_{N}^{2} \phi_{N}+\frac{1}{\rho c_{N}^{2}} \delta(p)=0, \quad N=1,2,
$$

where the wavenumber $k_{N}=\omega / c_{N}$ has been introduced. Since we have chosen the harmonic time dependence to be of the form $e^{-i \omega t}$, the outgoing wave solution of (52) in the $p$ coordinate is:

$$
\phi_{N}(p, \boldsymbol{n})=-\frac{e^{i k_{N}|p|}}{2 \rho i k_{N} c_{N}^{2}}
$$

so that

$$
\hat{\boldsymbol{G}}(p, \boldsymbol{n})=-\sum_{N=1}^{2} \frac{e^{i k_{N}|p|}}{2 \rho i k_{N} c_{N}^{2}} \boldsymbol{v}_{N} \otimes \boldsymbol{w}_{N}
$$

and

$$
\hat{\boldsymbol{G}}^{\prime}(p, \boldsymbol{n})=-\sum_{N=1}^{2} \frac{\operatorname{sgn}(p) e^{i k_{N}|p|}}{2 \rho c_{N}^{2}} \boldsymbol{v}_{N} \otimes \boldsymbol{w}_{N} .
$$

The antitransform of equation (54) leads to

$$
\boldsymbol{G}(\boldsymbol{x})=-\frac{1}{4 \pi^{2}} \sum_{N=1}^{2} \int_{|\boldsymbol{n}|=1} \int_{-\infty}^{+\infty} \frac{\operatorname{sgn}(p) e^{i k_{N}|p|}}{2 \rho c_{N}^{2}(\boldsymbol{n} \cdot \boldsymbol{x}-p)} \boldsymbol{v}_{N} \otimes \boldsymbol{w}_{N} d p d s
$$

The integral in the variable $p$ can be evaluated in the way shown in Appendix B, so that, employing the cosine and sine integral functions

$$
\operatorname{Ci}(z)=\int_{+\infty}^{z} \frac{\cos t}{t} d t, \quad|\arg z|<\pi \quad \text { and } \quad \operatorname{Si}(z)=\int_{0}^{z} \frac{\sin t}{t} d t
$$


the Green's function can be finally written in the form

$$
\begin{aligned}
\boldsymbol{G}(\boldsymbol{x})=-\frac{1}{8 \pi^{2}} \sum_{N=1}^{2} \int_{|\boldsymbol{n}|=1}\left[2 \cos \left(k_{N} \boldsymbol{n} \cdot \boldsymbol{x}\right) \operatorname{Ci}\left(k_{N}|\boldsymbol{n} \cdot \boldsymbol{x}|\right)\right. \\
\left.+2 \sin \left(k_{N} \boldsymbol{n} \cdot \boldsymbol{x}\right) \operatorname{Si}\left(k_{N} \boldsymbol{n} \cdot \boldsymbol{x}\right)-i \pi \cos \left(k_{N} \boldsymbol{n} \cdot \boldsymbol{x}\right)\right] \frac{\boldsymbol{v}_{N} \otimes \boldsymbol{w}_{N}}{\rho c_{N}^{2}} d s .
\end{aligned}
$$

We introduce polar coordinates so that the position vector $\boldsymbol{x}$ has modulus $r=|\boldsymbol{x}|$ and is inclined at angle $\theta$ to the $x_{1}$-axis. Taking the unit vector $\boldsymbol{n}$ inclined at $\alpha+\theta$ with respect to the $x_{1}$-axis (so that $\alpha$ is the angle between $\boldsymbol{x}$ and $\boldsymbol{n}$ ) and noting that $\cos (\cdot) \mathrm{Ci}(\cdot)$ and $\sin (\cdot) \operatorname{Si}(\cdot)$ are even functions, we can re-write eqn. (58) as

$$
\begin{aligned}
\boldsymbol{G}(\boldsymbol{x})= & -\frac{1}{8 \pi^{2}} \sum_{N=1}^{2} \int_{0}^{2 \pi}\left[2 \cos \left(r k_{N}|\cos \alpha|\right) \operatorname{Ci}\left(r k_{N}|\cos \alpha|\right)\right. \\
& \left.+2 \sin \left(r k_{N}|\cos \alpha|\right) \operatorname{Si}\left(r k_{N}|\cos \alpha|\right)-i \pi \cos \left(r k_{N}|\cos \alpha|\right)\right] \frac{\boldsymbol{v}_{N} \otimes \boldsymbol{w}_{N}}{\rho c_{N}^{2}} d \alpha
\end{aligned}
$$

where $k_{N}, \boldsymbol{v}_{N}, \boldsymbol{w}_{N}$ and $c_{N}^{2}$ depend on $\alpha+\theta$.

The acoustic tensor is a periodic function of $\alpha$ with period $\pi$ since

$$
\rho A_{i k}(\boldsymbol{n})=\mathbb{C}_{i 1 k 1} n_{1}^{2}+\left(\mathbb{C}_{i 1 k 2}+\mathbb{C}_{i 2 k 1}\right) n_{1} n_{2}+\mathbb{C}_{i 2 k 2} n_{2}^{2},
$$

where $n_{1}=\cos (\alpha+\theta)$ and $n_{2}=\sin (\alpha+\theta)$, and also $c_{N}, k_{N}, \boldsymbol{v}_{N}$, and $\boldsymbol{w}_{N}$ are periodic functions of $\alpha$ with the same period. It follows that the integrand in eqn. (59) is $\pi-$ periodic. Therefore,

the two-dimensional, time-harmonic Green's function corresponding to a generic, completely non-symmetric constitutive fourth-order tensor, relating the increment of the first Piola-Kirchhoff stress to the deformation gradient increment, eqn. (10), can be written in the form

$$
\begin{aligned}
\boldsymbol{G}(\boldsymbol{x})= & -\frac{1}{4 \pi^{2}} \sum_{N=1}^{2} \int_{0}^{\pi}\left[2 \cos \left(r k_{N}|\cos \alpha|\right) \operatorname{Ci}\left(r k_{N}|\cos \alpha|\right)\right. \\
& \left.+2 \sin \left(r k_{N}|\cos \alpha|\right) \operatorname{Si}\left(r k_{N}|\cos \alpha|\right)-i \pi \cos \left(r k_{N}|\cos \alpha|\right)\right] \frac{\boldsymbol{v}_{N} \otimes \boldsymbol{w}_{N}}{\rho c_{N}^{2}} d \alpha,
\end{aligned}
$$

where $k_{N}=\omega / c_{N}$ and $c_{N}^{2}$ are the eigenvalues of the acoustic tensor $\boldsymbol{A}$, eqn. (31) with corresponding left and right eigenvectors $\boldsymbol{w}_{N}$ and $\boldsymbol{v}_{N}$, all quantities depending on $\boldsymbol{n}$, which means on $\alpha+\theta$.

It can be noted that the integrand in eqn. (61) displays a logarithmic singularity at $r=0$ and $\alpha=\pi / 2$, since (Lebedev, 1965)

$$
\operatorname{Ci}(z)=\gamma+\log z-\int_{0}^{z} \frac{1-\cos t}{t} d t, \quad|\arg z|<\pi
$$

where $\gamma$ is Euler's constant. 


\section{A dynamical interpretation of flutter instability}

The dynamical interpretation of flutter instability will be achieved following the approach introduced by Bigoni and Capuani $(2002 ; 2005)$, so that the Green's function is employed to provide a dynamical perturbation to be superimposed upon a given state of equilibrium of a homogeneously deformed material. Several plots of Green's tensor components will be presented, so that a preliminary normalization of the Green's tensor and a study of the involved non-dimensional parameters becomes instrumental. In particular, introducing an arbitrary characteristic length $a$ and consequently the dimensionless spatial variable $\overline{\boldsymbol{x}}=\boldsymbol{x} / a$, making use of the property

$$
\delta(a \overline{\boldsymbol{x}})=\frac{1}{a^{2}} \delta(\overline{\boldsymbol{x}}), \quad \overline{\boldsymbol{x}} \in \mathbf{R}^{2},
$$

eqn. (39) can be rewritten as

$$
\overline{\mathbb{C}}_{i j k l} \frac{\partial^{2} \bar{G}_{k q}(\overline{\boldsymbol{x}})}{\partial \bar{x}_{j} \partial \bar{x}_{l}}+\bar{\omega}^{2} \bar{G}_{i q}(\overline{\boldsymbol{x}})+\delta_{i q} \delta(\overline{\boldsymbol{x}})=0, \quad \overline{\boldsymbol{x}} \in \mathbf{R}^{2}
$$

where

$$
\overline{\mathbb{C}}_{i j k l}=\frac{\mathbb{C}_{i j k l}}{\mu}, \quad \bar{\omega}=a \sqrt{\frac{\rho}{\mu}} \omega .
$$

Thus, a dimensionless version of the Green's tensor (158) reads

$$
\begin{aligned}
\overline{\boldsymbol{G}}(\overline{\boldsymbol{x}})=- & \frac{1}{8 \pi^{2}} \sum_{N=1}^{2} \int_{|\boldsymbol{n}|=1}\left[2 \cos \left(\bar{k}_{N} \boldsymbol{n} \cdot \overline{\boldsymbol{x}}\right) \operatorname{Ci}\left(\bar{k}_{N}|\boldsymbol{n} \cdot \overline{\boldsymbol{x}}|\right)\right. \\
& \left.+2 \sin \left(\bar{k}_{N} \boldsymbol{n} \cdot \overline{\boldsymbol{x}}\right) \operatorname{Si}\left(\bar{k}_{N} \boldsymbol{n} \cdot \overline{\boldsymbol{x}}\right)-i \pi \cos \left(\bar{k}_{N} \boldsymbol{n} \cdot \overline{\boldsymbol{x}}\right)\right] \frac{\boldsymbol{v}_{N} \otimes \boldsymbol{w}_{N}}{\bar{c}_{N}^{2}} d s
\end{aligned}
$$

where

$$
\bar{k}_{N}=a k_{N}=\frac{\bar{\omega}}{\bar{c}_{N}}, \quad \bar{c}_{N}=\sqrt{\frac{\rho}{\mu}} c_{N},
$$

so that $\bar{c}_{N}^{2}$ are the eigenvalues of the dimensionless acoustic tensor $\overline{\boldsymbol{A}}=\rho \boldsymbol{A} / \mu$.

\subsection{Effects of flutter instability on Green's tensor}

The behaviour of the Green's function, eqn. (61), is briefly analyzed here, outside and inside the flutter region. As a reference, we consider Case 3 shown in Fig. 4, in which the material is subject to the radial stress path corresponding to $\theta_{L}=0$ in Fig. 2 and the direction of the axis of elastic symmetry is taken inclined at $\theta_{\sigma}=45^{\circ}$ with respect to the principal stress direction $\boldsymbol{k}_{1}$. The employed material parameters are $\lambda / \mu=1, \hat{b}=80^{\circ}$, $\psi=30^{\circ}$, and $\chi=0^{\circ}$. The dimensionless Green's tensor components have been computed 
for $\bar{\omega}=1$ and for several values of the plastic modulus $H / \mu$, including the values 3.53, and 1.5. These correspond, respectively, to situations near and inside the flutter region (see Fig. (4), but still in a situation where the constitutive operator is positive definite. The values of the components are plotted in Fig. 5 as functions of the distance from the singularity along a radial line inclined at $-45^{\circ}$ with respect to the $x_{1}$ axis, normalized through division by $a$. The real (imaginary) parts of the Green's function components are plotted left (right) in the figure, the plots having been obtained starting from $x_{1}=1 / 10$ to exclude the singularity (in the real components of the Green's tensor).

Commenting on the results, first, we note from the figure that the Green's tensor is not symmetric (since the acoustic tensor is not), so that $G_{12} \neq G_{21}$.

Second, results referring to values of plastic modulus $H / \mu$ higher than 3.53 and up to 7 , not reported here for conciseness, produce curves practically coincident to those pertaining to $H / \mu=3.53$; we can therefore conclude that there is not much difference between the situations in which the material is far from and very near to the flutter region. This feature has been confirmed by us with several calculations (not reported here) and distinguishes flutter from shear banding, the latter becoming already visible when the condition of loss of ellipticity is approached from the interior of the elliptic range (Bigoni and Capuani, 2002; 2005).

Third, a blow-up of the solution with the space variable, clearly visible in all components of the Green's tensor is the characteristic feature of instability inside the flutter region, $H / \mu=1.5$. This blow-up is similar to that evidenced by Bigoni and Willis (1994), but in a constitutive setting including viscosity, which is now absent.

It becomes evident that further exploration of flutter instability requires plotting of incremental displacement maps. These are obtained below employing a perturbation in the form of a pulsating dipole.

\subsection{Effects of flutter instability revealed by a perturbing dipole}

The singular solution previously obtained, eqn. (61), can be used to analyze the effects of a perturbation superimposed upon a given homogeneous deformation of an infinite body. We follow here Bigoni and Capuani (2005) considering the simplest self-equilibrated perturbation in terms of a dipole: two equal and opposite pulsating forces of unit amplitude, taken at a distance $2 a$ apart, along a line inclined at $\beta=45^{\circ}$ with respect to the $x_{1}$-axis, see Fig. 6.

For this loading system, the level sets of the real part (left in the figures) and the imaginary part (right in the figures) of the components $u_{1}$ (first and third parts from the top of the figure) and $u_{2}$ (second and fourth parts from the top of the figure) of incremental displacements have been computed and plotted in Figs. 17 12. The two upper parts of all the figures refer to a situation far from flutter instability, whereas the two lower parts refer to a situation of flutter, well inside the region of instability. 


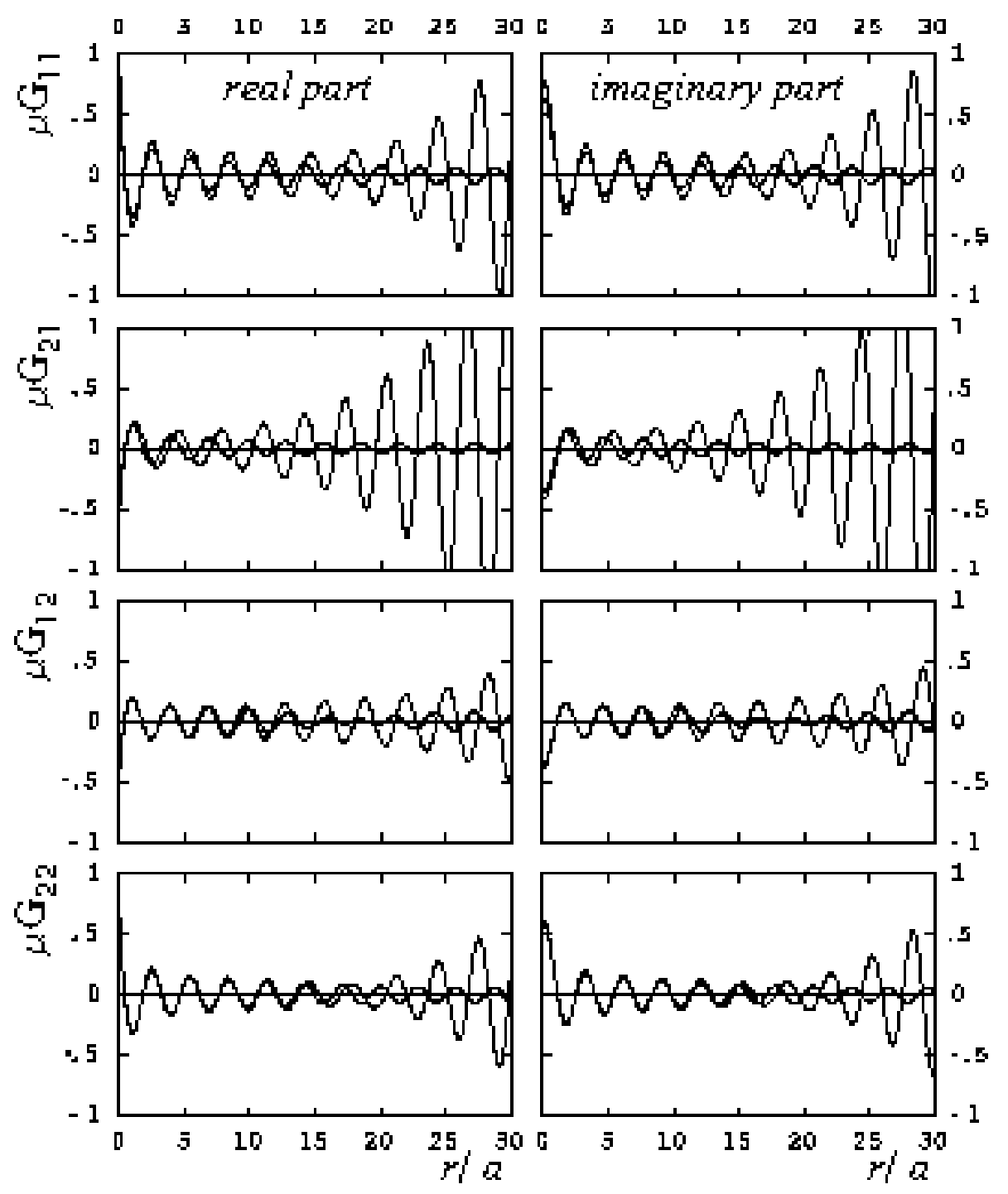

Figure 5: Dimensionless Green's tensor components (real part left, imaginary part right in the figure) along a radial line inclined at $-45^{\circ}$ with respect to $x_{1}$-axis, for Case 3 of Fig. 4 and $\bar{\omega}=1$. Two values of the plastic modulus $H / \mu=\{3.53,1.5\}$ are considered, corresponding, respectively, to situations near and inside the flutter region. The blow-up of all components of the Green's tensor is evident in the flutter region, $H / \mu=1.5$. 


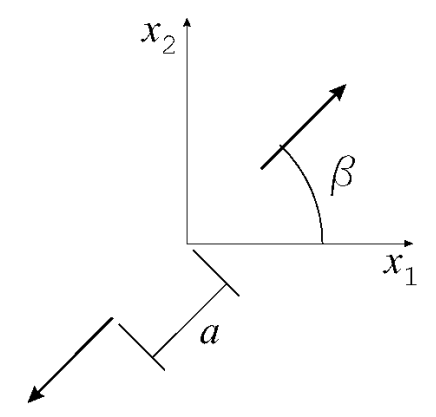

Figure 6: Geometry of the time-harmonic pulsating perturbing dipole.

The following parameters have been selected to be equal for all figures:

$$
\lambda / \mu=1, \quad \hat{b}=80^{\circ}, \quad \psi=30^{\circ}, \quad \chi=0^{\circ} .
$$

Moreover, Figs. 710 refer to the same nondimensional frequency parameter $\bar{\omega}=1$, whereas the effect of frequency is explored in Figs. 11] and 12, pertaining respectively to $\bar{\omega}=2$ and $1 / 2$ and corresponding to the same parameters employed in Fig. 8. All components of incremental displacements have been plotted for the nondimensional coordinates $x_{1} / a$ and $x_{2} / a$ ranging between -25 and 25, with the exception of Fig. 10, where this range has been extended to -50 and 50 to help visualization of the blowing-up typical of flutter.

The differences between Figs. 70 10 lie in the choice of different stress states expressed in terms of $\theta_{L}$ and anisotropy direction $\theta_{\sigma}$. In particular:

- Fig. 7 refers to $H / \mu=3$ (two upper parts), $H / \mu=0.32$ (two lower parts) and to Case 1 of Fig. 过, where $\theta_{L}=0^{\circ}$ and $\theta_{\sigma}=15^{\circ}$;

- Fig. 8 refers to $H / \mu=2$ (two upper parts), $H / \mu=0.25$ (two lower parts) and to Case 2 of Fig. 目, where $\theta_{L}=30^{\circ}$ and $\theta_{\sigma}=30^{\circ}$;

- Fig. 9 refers to $H / \mu=4$ (two upper parts), $H / \mu=1.5$ (two lower parts) and to Case 3 of Fig. 4, where $\theta_{L}=0^{\circ}$ and $\theta_{\sigma}=45^{\circ}$;

- Fig. 10 refers to $H / \mu=4$ (two upper parts), $H / \mu=1.9$ (two lower parts) and to Case 4 of Fig. 过, where $\theta_{L}=0^{\circ}$ and $\theta_{\sigma}=60^{\circ}$.

Note that the values of the plastic modulus selected for the examples are all higher than the critical values for loss of ellipticity [see the values listed in (27)], so that shear

\footnotetext{
${ }^{7}$ More precisely, all the considered plastic moduli are higher than the critical values for loss of strong ellipticity (Bigoni, 2000).
} 

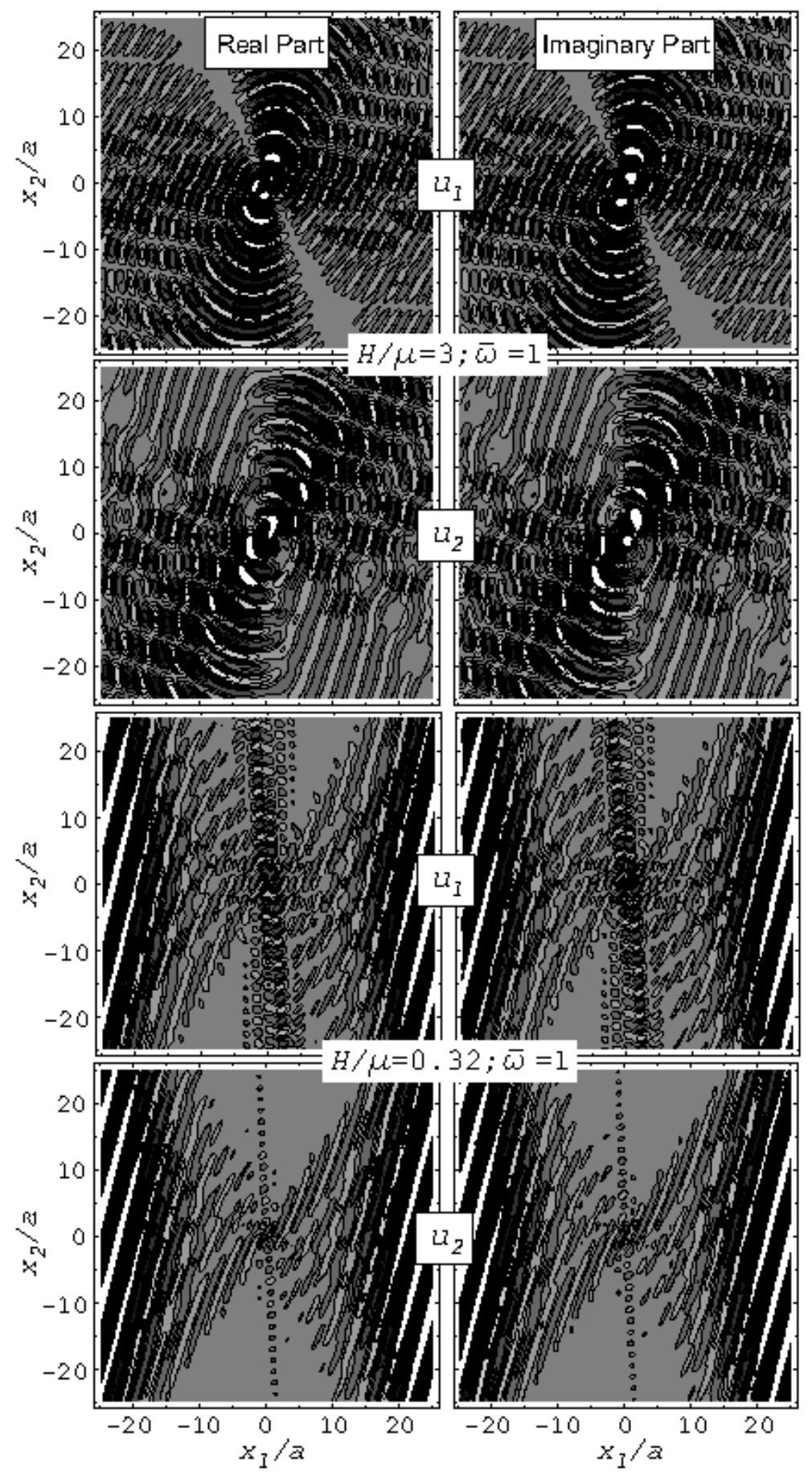

Figure 7: Level sets of the real (left) and imaginary (right) parts of the components of incremental displacements $\left(u_{1}\right.$ first and third parts from the top, $u_{2}$ second and fourth parts) for a dipole inclined at $\beta=45^{\circ}$, far from (upper two parts, $H / \mu=3$ ) and inside (lower two parts, $H / \mu=0.32$ ) the flutter

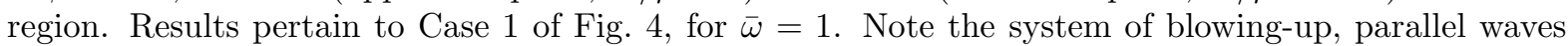
revealing the effect of flutter. 

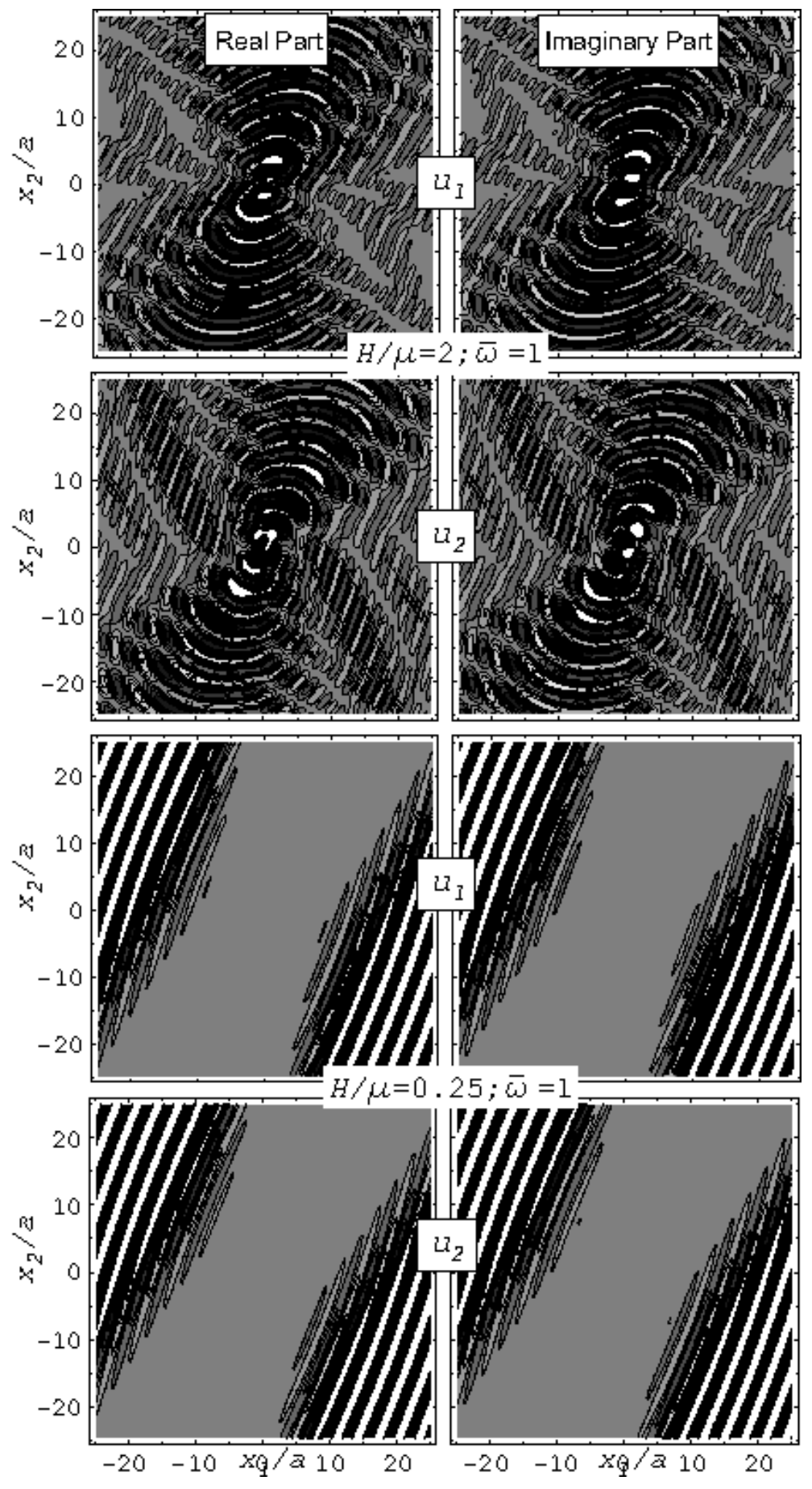

Figure 8: Level sets of the real (left) and imaginary (right) parts of (the modulus of) incremental displacements for a dipole inclined at $\beta=45^{\circ}$, far from (upper part, $H / \mu=2$ ) and inside (lower part,

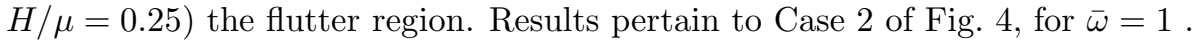


bands are excluded. However, all the values of $H / \mu$ corresponding to situations far from flutter and the two values 1.5 and 1.9 lie in the zone of positive definiteness of the constitutive operator, while the two values 0.25 and 0.32 have been selected outside this region [see the values listed in (27)].

It can be observed from the upper parts of Figs. 710 (referring to a non-flutter situation) that the displacement maps are typical of an anisotropic material, since $45^{\circ}-$ symmetry is not in evidence. Moreover, decay of the solution is appreciable, when the distance from the dipole increases. Now, considering the lower parts of the figures, the effects of flutter instability become self-evident. In particular, we may observe a growth of the solution in space, which tends to degenerate into a system of blowing-up, parallel plane waves. Results not reported here for brevity demonstrate that:

the inclination of the blowing-up plane waves is almost independent of the dipole inclination (angle $\beta$ in Fig. (6), so that it has to be considered a characteristic of the material, related to the particular stress state and constitutive features. We have observed that the inclination of the blowing-up waves corresponds to a value in the middle of the inclination fan of flutter (see Fig. 4).

In particular, the inclinations of the plane waves at a sufficient distance from the dipole, are different in Figs. 7,[10, but correspond to the mean value of flutter direction fan visible in Fig. 4 at the analyzed $H / \mu$ values. On the other hand, the same inclinations are found for figures Figs. 8 and 11 and 12 , since these cases differ only in the nondimensional frequency parameter $\bar{\omega}$, which influences only the spacing of the blowing-up waves.

As far as the effects of varying the nondimensional frequency parameter $\bar{\omega}$ are concerned (see Figs. 11] and 12, referring to the same material parameters as in Fig. 8, but with $\bar{\omega}=\{1,2,1 / 2\}$ ), we see that an increase in the frequency yields a narrowing of the distance between blowing-up plane waves. Moreover, increase in frequency gives rise to the 'shadowing' effect already noted by Bigoni and Capuani (2005) for shear bands.

Compared to the shear bands analyzed by Bigoni and Capuani (2002; 2005), we may observe that these are already revealed when the boundary of the region of ellipticity is approached from the inside, while flutter remains undetected. Beside this difference, there are however many similarities between the two phenomena: first of all, shear bands tend to blow-up in space as the boundary of instability is approached, and extend from a perturbation to infinity, outside the elliptic range. Second, shear bands also tend to degenerate into families of plane waves parallel to a specific direction. Third, the signals tend to focus along well defined patterns, both for shear bands and for flutter. Note however, that flutter instability may occur much earlier than shear banding in a deformation process; moreover, waves near the loss of ellipticity threshold tend to blowup along the shear bands but, in contrast to flutter, they tend to decay in the parallel direction.

As a conclusion, we remark that flutter instability yields a self-organization of dynamic disturbances along well-defined and blowing-up parallel waves, having inclinations 
corresponding to the mean value of the inclinations for which flutter is possible at the considered constitutive setting and stress state.

From the mechanical point of view, our results suggest that flutter yields a 'layering' of deformation patterns, with an inclination corresponding to the flutter direction, a spacing related to the frequency of the perturbing agency, and possibly occurring early in a plastic deformation process.

\section{Conclusions}

Following the approach to material instabilities proposed by Bigoni and Capuani (2002; 2005), flutter instability in a continuous elastoplastic medium has been investigated, by finding the dynamic, time-harmonic Green's function for the loading branch of a fully unsymmetric tangent constitutive operator, embodying features typical of the behaviour of granular materials. For this material, flutter instability may occur when the constitutive operator is positive definite (so that the solution of the rate infinitesimal problem is unique and shear bands are excluded), while two eigenvalues of the acoustic tensor are complex conjugate. Our results provide the first interpretation of flutter instability, which is shown to correspond to a dynamical instability growing in space and self-organizing into plane waves with normals lying in the fan corresponding to the complex eigenvalues of the acoustic tensor and yielding a sort of 'layering' of unstable deformation patterns, showing some similarity to shear band instability. The rate of growth of the solutions displayed here increases with the frequency that is assumed. This demonstrates dynamical ill-posedness of the governing equations of motion in the general transient case and implies a need that is physical as well as mathematical for the admission of some appropriate rate-dependence into the constitutive model, to remove the flutter effect at high frequencies. Although no such mechanism is built into the present analysis (the tangent moduli would become functions of $\omega$ but this is in any case fixed), and other mechanisms not accounted for (such as for instance the possibility of elastic unloading and material viscosity) may change some of our conclusions, we believe that the emergence of the layered structures that we have found may find future experimental validation.

\section{Acknowledgments}

Financial support of MURST-Cofin 2004 (Microstructural problems and models: applications in structural and civil engineering) is gratefully acknowledged. 

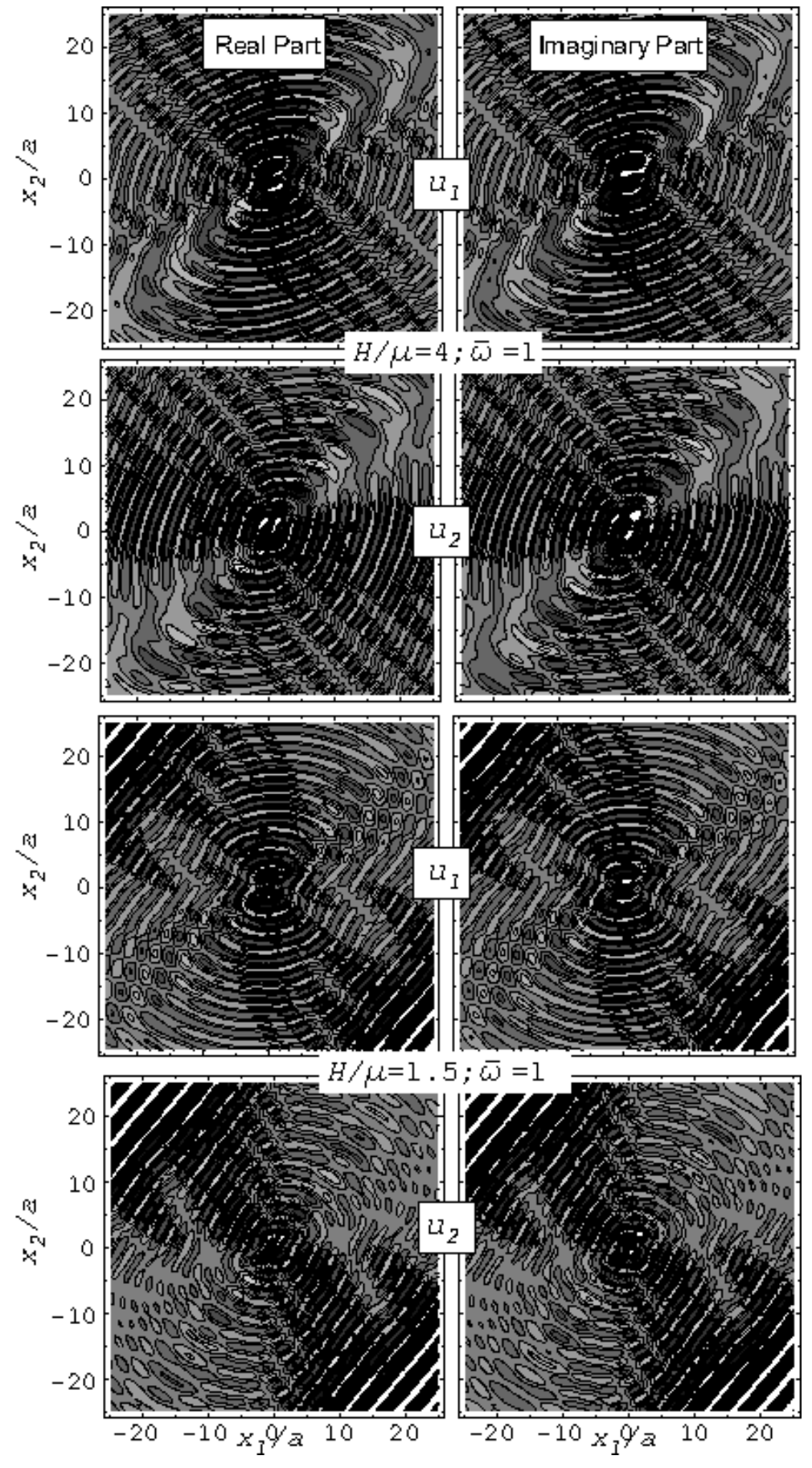

Figure 9: Level sets of the real (left) and imaginary (right) parts of (the modulus of) incremental displacements for a dipole inclined at $\beta=45^{\circ}$, far from (upper part, $H / \mu=4$ ) and inside (lower part, $H / \mu=1.5)$ the flutter region. Results pertain to Case 3 of Fig. 目 for $\bar{\omega}=1$. 

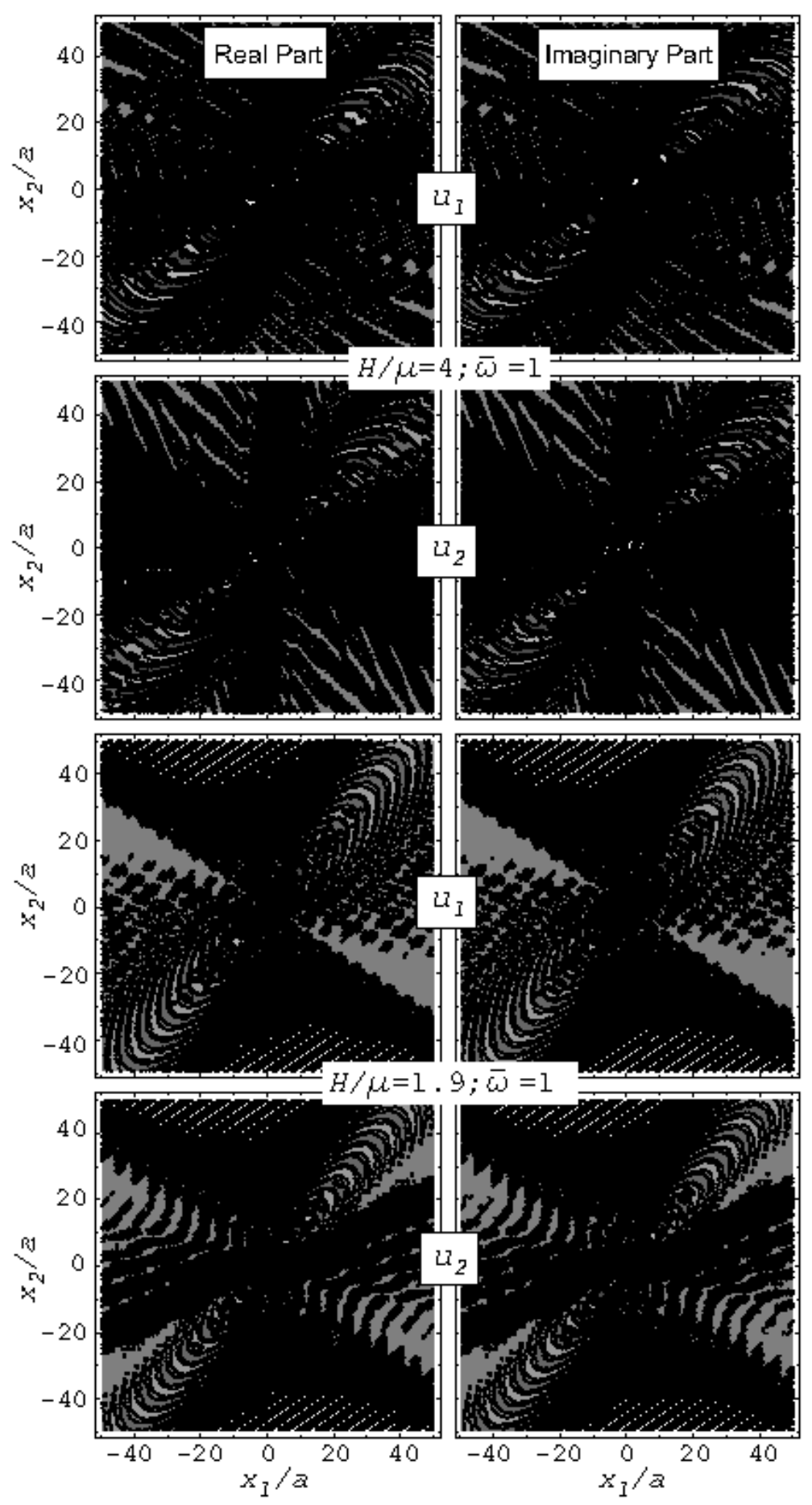

Figure 10: Level sets of the real (left) and imaginary (right) parts of (the modulus of) incremental displacements for a dipole inclined at $\beta=45^{\circ}$, far from (upper part, $H / \mu=4$ ) and inside (lower part, $H / \mu=1.9$ ) the flutter region. Results pertain to Case 4 of Fig. 固 for $\bar{\omega}=1$. 

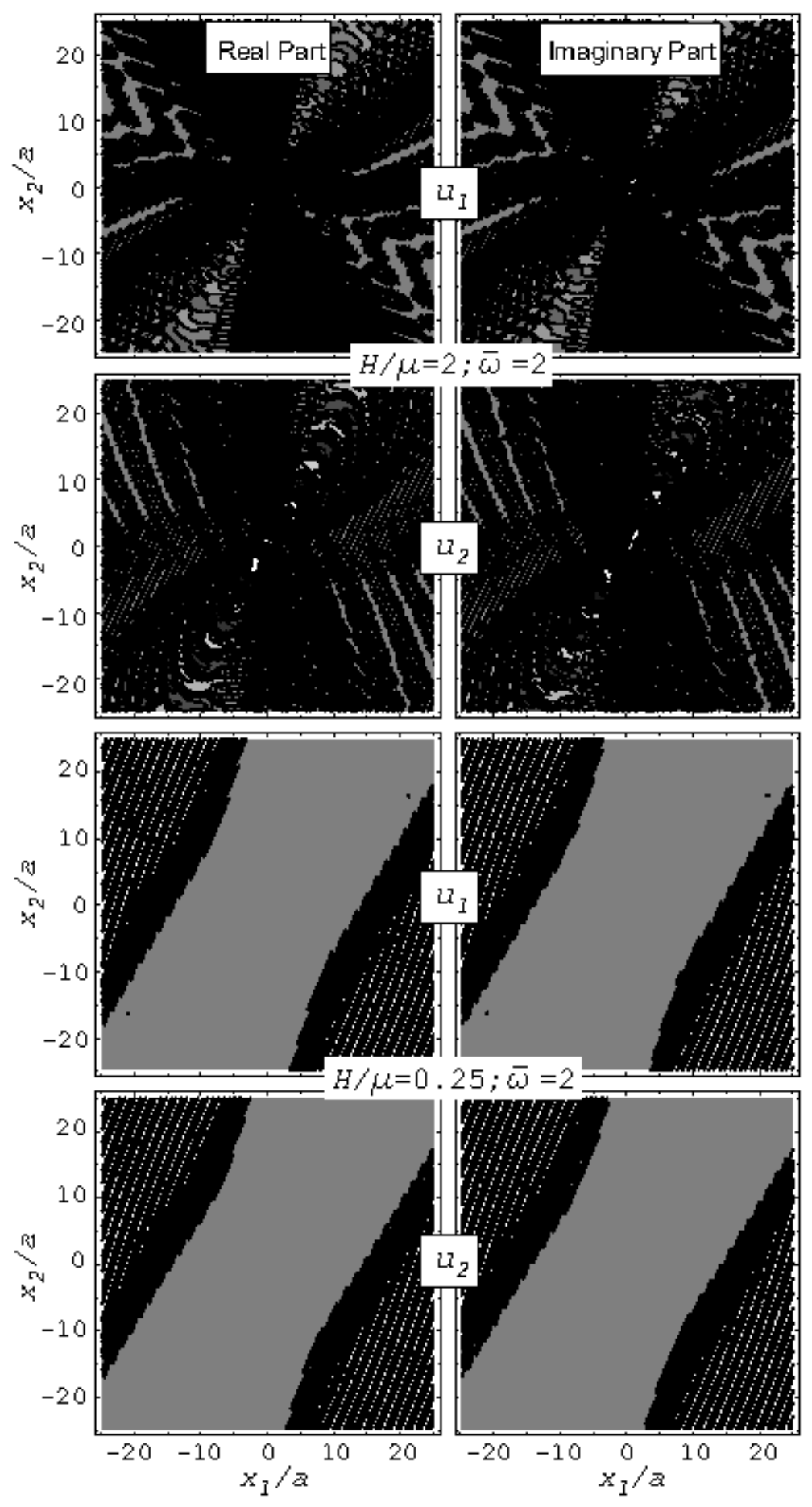

Figure 11: As for Fig. 8 , but with $\bar{\omega}=2$. 

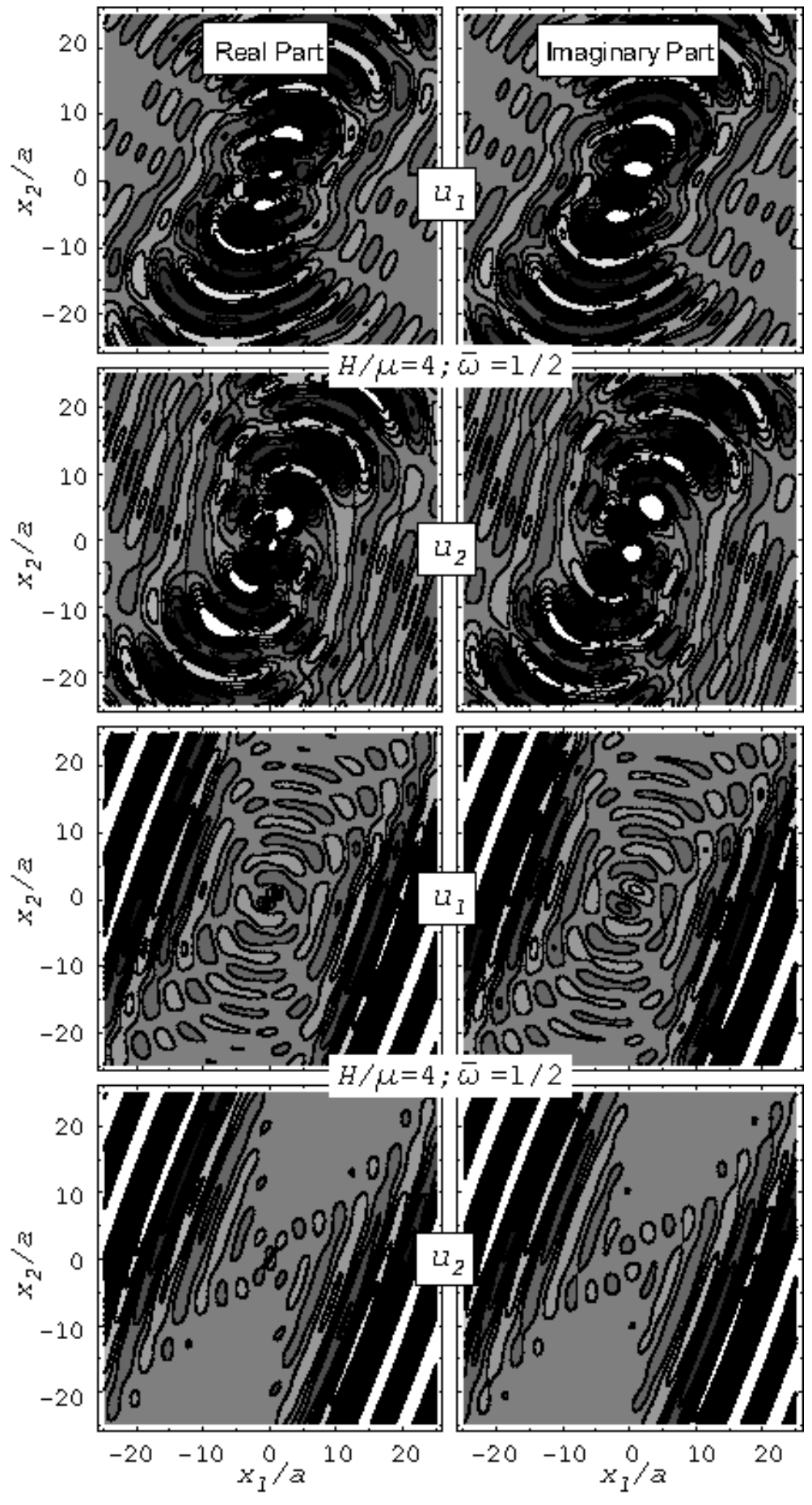

Figure 12: As for Fig. 8 , but with $\bar{\omega}=1 / 2$. 


\section{References}

[1] Bertoldi, K., Brun, M., Bigoni, D., 2005. A new boundary element technique for elastoplastic solids. Int. J. Numer. Meth. Eng. 64, 877-906.

[2] Bigoni, D., 1995. On flutter instability in elastoplastic constitutive models. Int. J. Solids Struct. 32, 3167-3189.

[3] Bigoni, D., 2000. Bifurcation and instability of nonassociative elasticplastic solids. In: Petryk, H. (Ed.), Material Instabilities in Elastic and Plastic Solids, CISM Lecture Notes No. 414, Springer-Verlag, WienNew York, pp. 1-52.

[4] Bigoni, D., Capuani, D., 2002. Green's function for incremental nonlinear elasticity: shear bands and boundary integral formulation. J. Mech. Phys. Solids 50, 471-500.

[5] Bigoni, D., Capuani, D., 2005. Time-harmonic Green's function and boundary integral formulation for incremental nonlinear elasticity: dynamics of wave patterns and shear bands. J. Mech. Phys. Solids 53, 1163-1187.

[6] Bigoni, D., Loret, B., 1999. Effects of elastic anisotropy on strain localization and flutter instability in plastic solids. J. Mech. Phys. Solids 47, 1409-1436.

[7] Bigoni, D., Loret, B., Radi, E., 2000. Localization of deformation in plane elasticplastic solids with anisotropic elasticity. J. Mech. Phys. Solids 48, 1441-1466.

[8] Bigoni, D., Petryk, H., 2002. A note on divergence and flutter instabilities in elasticplastic materials. Int. J. Solids Struct. 39, 911-926.

[9] Bigoni, D., Willis, J.R., 1994. A dynamical interpretation of flutter instability. In: Chambon, R., Desrues, J. and Vardoulakis, I. (Eds.), Localisation and Bifurcation of Rocks and Soils, Rotterdam: A.A. Balkema Scientific Publishers, pp. 51-58.

[10] Bigoni, D., Zaccaria, D., 1994. On eigenvalues of the acoustic tensor in elastoplasticity. Eur. J. Mech. A-Solids. 13, 621-638.

[11] Lebedev, N.N. 1965. Special functions and their applications, Prentice-Hall, Inc..

[12] Loret, B., Simões, F.M.F., Martins, J.A.C., 2000. Flutter instability and illposedness in solids and fluid-saturated porous media. In: Petryk, H. (Ed.), Material Instabilities in Elastic and Plastic Solids, CISM Lecture Notes No. 414, SpringerVerlag, WienNew York, pp. 109-207.

[13] Rice, J. R., 1977. The localization of plastic deformation. In: Koiter, W.T., (Ed.), Theoretical and Applied Mechanics. Amsterdam:North-Holland, pp. 207-220. 
[14] Simões, F.M.F., 1997. Instabilities in non-associated problems of solid mechanics. Ph.D. Thesis, Technical University of Lisbon, in Portuguese.

[15] Willis, J.R., 1991. Inclusions and cracks in constrained anisotropic media. In: Wu, J.J., Ting, T.C.T. and Barnett, D.M. (Eds.), Modern Theory of Anisotropic Elasticity and Applications. SIAM, Philadelphia, pp. 87-102. 


\section{APPENDIX A. Green's function obtained via plane wave expansion.}

The Green's function (58) is obtained here for completeness using the plane wave expansion technique employed by Bigoni and Capuani (2005). The plane wave expansion of the $\delta$ function and of the Green's tensor $\boldsymbol{G}(\boldsymbol{x})$ are, respectively,

$$
\delta(\boldsymbol{x})=-\frac{1}{4 \pi^{2}} \int_{|\boldsymbol{n}|=1} \frac{1}{(\boldsymbol{n} \cdot \boldsymbol{x})^{2}} d s, \quad \boldsymbol{G}(\boldsymbol{x})=-\frac{1}{4 \pi^{2}} \int_{|\boldsymbol{n}|=1} \tilde{\boldsymbol{G}}(\boldsymbol{n} \cdot \boldsymbol{x}) d s,
$$

where $\boldsymbol{n}$ is a unit vector, so that the plane wave expansion of eqn. (39) leads to

$$
\mathbb{C}_{i j k l} n_{j} n_{l} \tilde{G}_{k q}^{\prime \prime}(\xi)+\rho \omega^{2} \tilde{G}_{i q}(\xi)+\frac{\delta_{i q}}{\xi^{2}}=0,
$$

where $\xi=\boldsymbol{n} \cdot \boldsymbol{x}$. In this equation the acoustic tensor can be easily recognized, $A_{i k}=$ $\mathbb{C}_{i j k l} n_{j} n_{l}$, so that we get

$$
\boldsymbol{A}(\boldsymbol{n}) \tilde{\boldsymbol{G}}^{\prime \prime}(\xi)+\omega^{2} \tilde{\boldsymbol{G}}(\xi)+\frac{1}{\rho \xi^{2}} \boldsymbol{I}=\mathbf{0}
$$

Writing now the analogue of the representation (50), namely,

$$
\tilde{\boldsymbol{G}}(\xi)=\sum_{N=1}^{2} \phi_{N}(\xi) \boldsymbol{v}_{N} \otimes \boldsymbol{w}_{N}
$$

we transform eqn. (A.3) into the analogue of eqn. (51)

$$
\sum_{N=1}^{2}\left(c_{N}^{2} \phi_{N}^{\prime \prime}+\omega^{2} \phi_{N}+\frac{1}{\rho \xi^{2}}\right) \boldsymbol{v}_{N} \otimes \boldsymbol{w}_{N}=\mathbf{0}
$$

which is equivalent to the following uncoupled system of two equations, analogous to eqns. (52),

$$
\phi_{N}^{\prime \prime}+k_{N}^{2} \phi_{N}+\frac{1}{\rho c_{N}^{2}} \frac{1}{\xi^{2}}=0, \quad N=1,2
$$

where $k_{N}=\omega / c_{N}$.

The sole physically meaningful solution of the ordinary differential equation (A.6) is obtained by imposing the radiation condition, stating that the solution should include only outgoing waves. Since the harmonic time dependence has been selected in the form $e^{-i \omega t}$, the outgoing wave solution of (A.6) in the $\xi$ coordinate is:

$$
\begin{aligned}
\phi_{N}(\xi)=\frac{1}{2 \rho c_{N}^{2}}\left[2 \operatorname{Ci}\left(k_{N}|\xi|\right)\right. & \cos \left(k_{N} \xi\right) \\
+ & \left.2 \operatorname{Si}\left(k_{N} \xi\right) \sin \left(k_{N} \xi\right)-i \pi \cos \left(k_{N} \xi\right)\right] .
\end{aligned}
$$


Finally, a chain of substitutions, of eqn. (A.7) into eqn. (A.4) and finally into eqn.

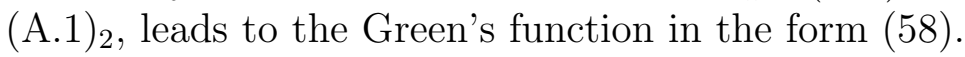

APPENDIX B. Evaluation of the integral in the variable $p$ in eqn. (56).

The integral in the variable $p$ appearing in eqn. (56) can be evaluated splitting the domain as follows

$$
\int_{-\infty}^{+\infty} \frac{\operatorname{sgn}(p) e^{i k_{N}|p|}}{\xi-p} d p=-\int_{-\infty}^{0} \frac{e^{-i k_{N} p}}{\xi-p} d p+\int_{0}^{+\infty} \frac{e^{i k_{N} p}}{\xi-p} d p
$$

so that we can treat the two integrals separately, namely

$$
-\int_{-\infty}^{0} \frac{e^{-i k_{N} p}}{\xi-p} d p=-e^{-i k_{N} \xi} \int_{k_{N} \xi}^{+\infty} \frac{e^{i q}}{q} d q
$$

where we have made the substitution $q=k_{N}(\xi-p)$, and

$$
\int_{0}^{+\infty} \frac{e^{i k_{N} p}}{\xi-p} d p=-e^{i k_{N} \xi} \int_{-k_{N} \xi}^{+\infty} \frac{e^{i q}}{q} d q
$$

where we have made the substitution $q=k_{N}(p-\xi)$. The two expressions (B.2) and (B.3) are used to get eqn. (58). 